\title{
A coupled non-Fickian model of a cardiovascular drug delivery system
}

\author{
J.A. Ferreira, J. Naghipoor $†$, and Paula de Oliveira ${ }^{\ddagger}$ \\ CMUC, Department of Mathematics, University of Coimbra, 3001-454, Coimbra, \\ Portugal
}

\begin{abstract}
A coupled non-Fickian model of a cardiovascular drug delivery system using a biodegradable drug eluting stent is proposed. The numerical results are obtained using an IMEX finite element method. The influence of vessel stiffness in the transport of drug eluted from the stent is analyzed. The results presented in this paper suggest new perspectives to adapt the drug delivery profile to the needs of the patient.

Keywords: Non-Fickian coupled model, cardiovascular drug delivery, drug eluting stent, numerical simulation.

Mathematics Subject Classification (2010): 65M60, 92-08.
\end{abstract}

\section{Introduction}

A stent is a device with the form of a mesh tube which is inserted into a natural body passage to expand its walls and to provide mechanical support for the wounded tissues. Even if stents are widely used in many medical specialities, its most common use is in vascular surgery to reduce stenosis, that is the narrowing of the arteries.

A Drug Eluting Stent (DES) is a stent that releases anti-proliferative drug into the arterial wall with a programmed pharmacokinetics. It consists of a metallic stent strut coated with a polymeric layer, that encapsulates a therapeutic drug, that will act to reduce smooth muscle cell growth and to prevent an inflammatory response which are the predominant causes of neointima proliferation and in-stent restenosis. Biodegradable polymers like polylactic acid (PLA) have become the materials of choice to coat stents while encapsulating the drug ([34]).

The vessel walls of the cardiovascular system are known to display a complex mechanical response under physiological conditions. Arterial stiffness is considered as an excellent indicator of cardiovascular morbidity and mortality in a large percentage of the population as referenced in [16]. The coronary artery is a complex structure mainly composed of three concentric layers: intima,

\footnotetext{
*ferreira@mat.uc.pt

†jahed.naghipoor@uni-weimar.de

† poliveir@mat.uc.pt
} 


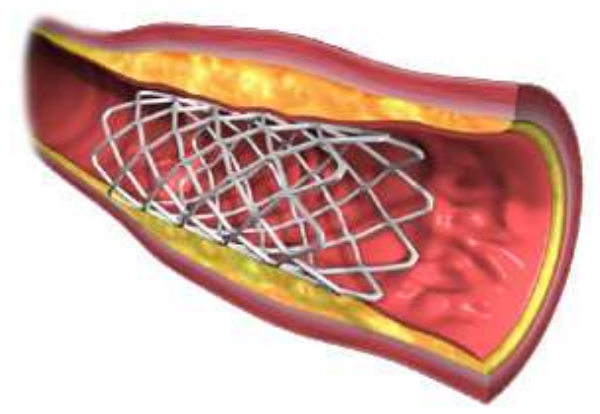

Figure 1: Detail of the stented artery (http://www.zmescience.com/research/bioreabsorbablestents-to-revolutionize-heart-surgeries).

media and adventitia. It mainly consists of elastin, which is responsible for the vessel elasticity, combined with collagen. Elastin and collagen are responsible for the viscoelastic properties of the vessel. These properties have been clearly demonstrated by laboratorial experiments like creep and relaxation tests $[11,24,37]$.

Due to the involvement of so many factors, prediction of drug release appears to be an important issue and mathematical models constitute key tools to design appropriate drug delivery systems. During the last years, a number of studies have proposed mathematical models for coupled drug delivery in the cardiovascular tissues. We refer without being exhaustive to $[2,4,14,19,22-29,40,41]$ as well as the comprehensive and the most recent review paper [20]. Most of these studies address the release of drug and its numerical behavior while the viscoelasticity of the vessel wall and the behaviour of the biodegradable materials are disregarded.

In this paper, we propose a non-Fickian coupled model for predicting the biodegradation of PLA as a drug carrier in the coated stent and the simultaneous release of the drug from the coating into the vessel wall. The effect of viscoelasticity of the vessel wall in the drug release is investigated using Maxwell-Wiechert model ([5]) and Fung's model ([11]).

To reduce the computational time, we perform the analysis of reaction-diffusion-convection of drug from stent coating into the arterial wall on a portion of the coronary artery. The influence of the geometry of the stent strut on drug release is considered negligible. Since the role of the therapeutic agent is to heal the artery after the implantation of DES, most of the computational studies in our work have focused on the transport of the drug into the arterial wall. In particular, we study the influence of the stiffness of the arterial wall on the drug release profile.

We assume that the arterial walls, as well as the stent coating, behave as a porous media with respect to the filtration of plasma and the transfer of molecules. So drug transfer in the arterial wall and in the stent coating is not only affected by diffusion but also depends on the advection induced by plasma filtration activated by physiological transmural pressure gradients. The Peclet number in our model is around 1 . Consequently both diffusion and convection terms must be taken into account in the transport process.

At low strains (physiological pressures), the media, the thickest tissue layer constituting the arterial wall, mainly determines its mechanical properties. Due to the high content of smooth muscle cells, compared to other layers, it is the media that is responsible for the viscoelastic behavior of the arterial wall ([37]). As reported in [21], the inclusion of adventitia has a negligible effect on cellular drug concentration in the media. So we simplify the complex multilayered structure of 
the arterial wall considering only the interaction of the stent with media. Consequently, the nonFickian reaction-diffusion-convection model is represented by a system of multidimensional partial differential equations defined in the media tissue and the coating of the stent. These equations describe the filtration of plasma, the diffusion, transport, and chemical reactions of PLA inside the stent coating as well as inside the arterial wall ([41]).

The outline of the paper is as follows: Section 2 is devoted to the description of the model and its initial, boundary and interface conditions. In Section 3 we briefly explain the mass behaviour of the materials following a phenomenological approach. Numerical simulations as well as a sensitivity analysis of the viscoelastic parameters are discussed in Section 4. In Section 5 some conclusions are presented. Mathematical results concerning the stability of the continuous and discrete models as well as the validation of the numerical model are presented in annexes $A$ to $C$.

\section{$2 \quad$ Description of the model}

We present in Section 2 a coupled model that describes the kinetics of the drug in the polymeric coating of the stent $\mathrm{S}$ and the vessel wall V. For a sake of clarity the equations that govern the transport of drug in the stent and the vessel wall are exhibited separately in subsections 2.1 and 2.2 respectively. The meaning and units of all variables are presented in Table 1.

\subsection{Mathematical modeling in the stent coating}

Let us consider a two dimensional domain obtained as a section of a three dimensional vessel geometry. Assuming the symmetry of the geometry we obtain a reduction of computational cost, by considering only a part of the section. The observation of OCT (Optical Coherence Tomography) images of diseased vessels of cardiovascular patients shows that the cross section of the lumen is not, in many cases, a perfect circle. In this sense the symmetry of the geometry is an academic assumption that will be improved in future studies. We introduce the two dimensional domain $S \subset \mathbb{R}^{2}$ which stands for the polymeric coating of the stent and $V \subset \mathbb{R}^{2}$ that represents the arterial wall. A schematic representation of the two dimensional domain used in this paper is shown in Figure 2.

When a DES is implanted in a vessel, the coated stent will be gradually covered by the neointima. The degree of embedding of the stent depends on the type of plaque in the arterial wall. When the plaque is lipidic, the stent is almost instantaneously embedded; in the case of a calcified plaque the complete embedding can occur after several months or even never occur ([14]). Between these two cases the depth of the embedding is defined by the proliferation rate of the neo-intima smooth muscle cells. For a sake of simplicity, we consider that the drug eluting stent is already inside of the arterial wall. This is a simplification with respect to the complex dynamics of tissue healing and regrowth that takes place after the implantation. However, since we are interested in a comparative study between different configurations of the physical parameters characterizing the stent as well as the viscoelastic properties of the arterial wall, the evolution of the wall around the stent can be initially neglected $([14,41])$. In future studies this aspect will be addressed. 


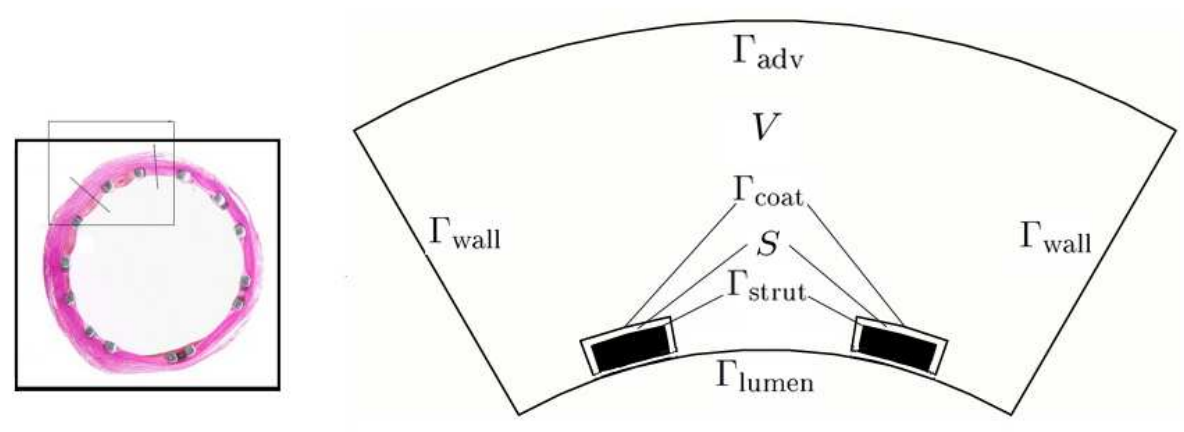

Figure 2: Drug eluting stent inside the vessel wall.

Three main phenomena explain the kinetics of the drug and the biodegradable polymer in the stent: chemical reactions, convection and diffusion.

\section{Chemical reactions}

Mathematical modeling of drug delivery, from a biodegradable coating into the arterial wall, is relatively complex when compared with modeling of drug release from a non-degradable polymer. In the case of a biodegradable coating, in addition to the physical mass transport process, the model has to account for the chemical processes involved in the biodegradation.

There are different approaches to describe reaction terms. Soares et al. ([36]) have proposed a multi-scale description of degradation and erosion combining the molecular description of scission with Fick's law of diffusion. In [30], a description of degradation reactions was coupled with drug transport and restenosis. The influence of the surrounding environment in the device (as $\mathrm{pH}$ value and cellular tissue reactions) in the degradation models was mentioned in [33]. In the present work we use the chemical reactions proposed in [29].

In the stent coating two reactions are responsible for the degradation of PLA into lactic acid and oligomers. In the first reaction, the hydrolysis of PLA occurs resulting in molecules with smaller molecular weights: oligomers (with molecular weight $M_{W}$ such that $2 \times 10^{4} \mathrm{~g} / \mathrm{mol} \leq M_{W} \leq$ $1.2 \times 10^{5} \mathrm{~g} / \mathrm{mol}$ ), lactic acid (with molecular weight satisfying $M_{W} \leq 2 \times 10^{4} \mathrm{~g} / \mathrm{mol}$ ). It is assumed that all these oligomers have similar diffusivities. The second reaction is the hydrolysis of the oligomers resulting in lactic acid. The lactic acid generated by this reaction has a catalytic effect on further degradation of the PLA. The rates of catalysis are represented in (3) by $\alpha$ and $\beta$.

Let $C_{1, S}$ and $C_{2, S}$ be the concentrations of plasma and PLA in the stent respectively. We note that by concentration of plasma we mean the concentration of water in the plasma. The concentration of oligomers in the stent is denoted by $C_{3, S}$ while $C_{4, S}$ and $C_{5, S}$ denote the concentrations of lactic acid and drug in the stent respectively. 


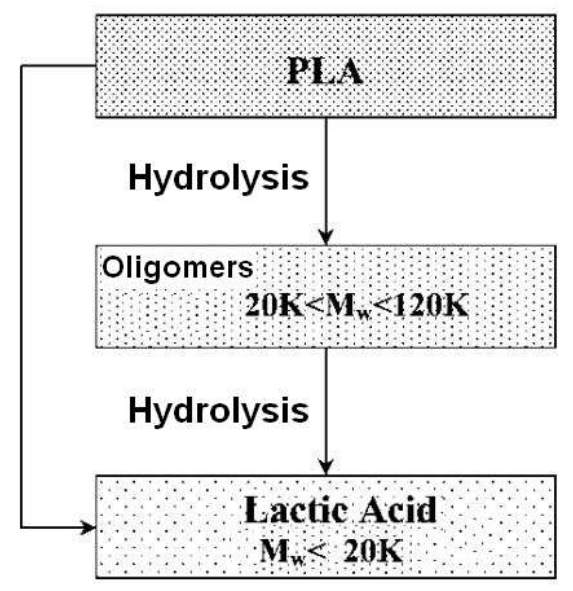

Figure 3: Schematic representation of the mathematical model for predicting degradation of PLA and drug release $([29])$.

The previously mentioned reactions are represented schematically by

$$
C_{1, S}+C_{2, S} \stackrel{\kappa_{1, S}}{\longrightarrow} C_{3, S}+C_{4, S}, \quad C_{1, S}+C_{3, S} \stackrel{\kappa_{2, S}}{\longrightarrow} C_{4, S},
$$

where $\kappa_{1, S}$ and $\kappa_{2, S}$ denote the reaction rates of the hydrolysis of PLA and oligomers in the stent (see Figure 3).

To simplify the definition of the reaction terms that affect the behaviour of each concentration in the stent, we introduce the notation $\mathcal{C}_{S}=\left(C_{m, S}\right)_{m=1, \ldots, 5}$.

The evolution in time and space of the concentrations depends on the type of chemical reaction involved: production or consumption. Let $F_{m, S}\left(\mathcal{C}_{S}\right), m=1, \ldots, 5$, represent the reaction terms to be considered in the evolution of the concentration in the stent. We adopt in what follows the reaction terms for the stent coating introduced in [29] and used in [9] which are defined by

$$
F_{m, S}\left(\mathcal{C}_{S}\right)= \begin{cases}-\sum_{i=1,2} \mathcal{F}_{i, S}\left(\mathcal{C}_{S}\right), & \mathrm{m}=1, \\ -\mathcal{F}_{1, S}\left(\mathcal{C}_{S}\right), & \mathrm{m}=2, \\ \sum_{i=1,2}(-1)^{i-1} \mathcal{F}_{i, S}\left(\mathcal{C}_{S}\right), & \mathrm{m}=3, \\ \sum_{i=1,2} \mathcal{F}_{i, S}\left(\mathcal{C}_{S}\right), & \mathrm{m}=4, \\ 0, & \mathrm{~m}=5 .\end{cases}
$$

$\operatorname{In}(2)$

$$
\begin{aligned}
& \mathcal{F}_{1, S}\left(\mathcal{C}_{S}\right)=\kappa_{1, S} C_{1, S} C_{2, S}\left(1+\alpha C_{4, S}\right), \\
& \mathcal{F}_{2, S}\left(\mathcal{C}_{S}\right)=\kappa_{2, S} C_{1, S} C_{3, S}\left(1+\beta C_{4, S}\right),
\end{aligned}
$$

where $\alpha, \beta$ stand for positive dimensional constants that quantify the extent of the auto-catalytic effect of lactic acid. This means that the lactic acid produced by reactions (1) acts as a catalyzer in the PLA degradation. The negative signs in (2) indicate the consumption of molecules while the positive signs indicate the production of molecules. For example, the reaction term for plasma 
$(m=1)$ in $(2)$ indicates that the fluid is consumed causing the degradation of PLA and the subsequent hydrolysis of oligomers.

The effect of the extracellular enzymes in the degradation process is neglected in this model. As mentioned in [31], the degradation rates measured "in vitro" are essentially the same as that measured "in vivo". So the major route of degradation for PLA is most likely via non-enzymatic hydrolysis. It is also assumed that drug do not react with PLA and its reaction products.

\section{Convection}

The transport of PLA, oligomers, lactic acid and drug in the stent coating occurs by diffusion and convection. The convection is caused by a pressure gradient in the fluid. Let $p_{S}$ represent the pressure in the stent while $u_{S}$ stands for the corresponding velocity. We also assume that the plasma is incompressible, which mathematically implies that the divergence of its velocity is zero, $\nabla \cdot u_{S}=0$. The behaviour of the plasma is described by Darcy's law. As the metallic part of the stent is impermeable we consider no flux of plasma across $\Gamma_{\text {strut }}$ which represents the interface between the stent coating and the metallic part of the stent (Figure 2). The velocity and the pressure in the stent coating satisfy then the following equations:

$$
\begin{cases}u_{S}=-\frac{k_{S}}{\mu_{S}} \nabla p_{S} & \text { in } S \\ \nabla \cdot u_{S}=0 & \text { in } S \\ u_{S} \cdot \eta_{S}=0 & \text { on } \Gamma_{\text {strut }}\end{cases}
$$

where $\eta_{S}$ represents the exterior unit normal.

The permeability $k_{S}$ depends on the properties of the medium and also on the concentrations of PLA, oligomers, lactic acid and drug in the stent coating. To simplify the model we assume that $k_{S}$ is constant. The viscosity $\mu_{S}$ depends on the chemical compounds present in the stent coating. To simplify, we also assume in what follows that the viscosity is constant.

\section{The complete model in the stent coating}

The reaction-convection-diffusion processes that take place in the stent coating are described by the following system of equations

$$
\frac{\partial C_{m, S}}{\partial t}=\nabla \cdot\left(D_{m, S} \nabla C_{m, S}-u_{S} C_{m, S}\right)+F_{m, S}\left(\mathcal{C}_{S}\right) \quad \text { in } S \times(0, T], m=1, \ldots, 5 .
$$

The diffusivities of the fluid, the oligomers, the lactic acid and the drug will vary during the degradation process [33]. We assume that the diffusion coefficient of each specie in the stent increases exponentially with the degradation of the PLA. In $[29,33]$ the expression

$$
D_{m, S}=D_{m, S}^{0} \mathrm{e}^{\theta_{m, S} \frac{C_{2, S}^{0}-C_{2, S}}{C_{2, S}^{0}}}, \quad m=1, \ldots, 5,
$$

was proposed where $D_{m, S}^{0}$ is the diffusion coefficient of the corresponding specie in the unhydrolyzed PLA, $C_{2, S}^{0}$ is the concentration of the unhydrolyzed polymer at $t=0$ and $\theta_{m, S}, m=1, \ldots, 5$, are some adimensional constants. 
At the initial time, we assume that the PLA and the drug are uniformly distributed. We also assume that at the initial time no degradation has occurred and consequently that there are neither oligomers nor lactic acid in the coating. The initial conditions in the coating are then given by

$$
\left\{\begin{array}{l}
C_{m, S}(0)=0, m=1,3,4, \\
C_{m, S}(0)=1, m=2,5 .
\end{array}\right.
$$

Here and in what follows we denote by $v(t)$ a function that depends on $x, y$ and $t$, that is for each $t, v(t): \bar{\Omega} \longrightarrow \mathbb{R}$, where $\bar{\Omega}$ represents $\bar{S}$ or $\bar{V}$.

As the metallic stent strut is impermeable to the drug and PLA degradation products that diffuse in coating stent, no mass flux passes through the boundary surface $\Gamma_{\text {strut. }}$. So

$$
J_{m, S} \cdot \eta_{S}=0, m=1, \ldots, 5
$$

where $J_{m, S}$ is the mass flux in the stent coating defined by

$$
J_{m, S}=-D_{m, S} \nabla C_{m, S}+u_{S} C_{m, S}, m=1, \ldots, 5 .
$$

The other boundary condition in $\Gamma_{\text {coat }}$ will be presented in Section 2.2 .

\subsection{Mathematical modeling of the arterial wall}

\section{Chemical reactions}

Let $C_{1, V}$ be the concentration of plasma in the vessel wall. The concentrations of oligomers and lactic acid in the vessel wall are denoted by $C_{3, V}$ and $C_{4, V}$ respectively. By $C_{5, V}$ we represent the concentration of drug in the vessel wall. As the PLA can not diffuse into the arterial wall ([29]), the only reaction in the arterial wall is the hydrolysis of the oligomers resulting in lactic acid which is represented schematically by

$$
C_{1, V}+C_{3, V} \stackrel{\kappa_{1, V}}{\longrightarrow} C_{4, V},
$$

where $\kappa_{1, V}$ denotes the reaction rate of the hydrolysis of oligomers in the arterial wall.

To simplify the presentation of the reaction terms that affect the behaviour of each concentration, we introduce $\mathcal{C}_{V}=\left(C_{m, V}\right)_{\substack{m=1, \ldots, 5, m \neq 2}}$

Considering reaction (10), $F_{m, V}\left(\mathcal{C}_{V}\right), m=1, \ldots, 5, m \neq 2$, are the reaction terms in the arterial wall, defined by

$$
F_{m, V}\left(\mathcal{C}_{V}\right)= \begin{cases}-\mathcal{F}_{1, V}\left(\mathcal{C}_{V}\right), & \mathrm{m}=1, \\ -\mathcal{F}_{1, V}\left(\mathcal{C}_{V}\right), & \mathrm{m}=3, \\ \mathcal{F}_{1, V}\left(\mathcal{C}_{V}\right), & \mathrm{m}=4, \\ 0, & \mathrm{~m}=5,\end{cases}
$$


where

$$
\mathcal{F}_{1, V}\left(\mathcal{C}_{V}\right)=\kappa_{1, V} C_{1, V} C_{3, V}\left(1+\gamma C_{4, V}\right)
$$

and $\gamma$ is a positive dimensional constant.

\section{Convection}

The transport of oligomers, lactic acid and drug in the vessel wall, occurs by diffusion and convection. The convection is caused by a pressure gradient in the fluid. Let $p_{V}$ and $u_{V}$ represent the pressure and velocity in the vessel wall respectively. We also assume that the plasma is incompressible $\left(\nabla \cdot u_{V}=0\right)$ and that its behaviour is described by Darcy's law.

To prescribe suitable boundary conditions in the arterial wall, we require that $u_{V} \cdot \eta_{V}=0$ on $\Gamma_{\text {wall }}$ for symmetry, where $\eta_{V}$ represents the exterior unit normal. Moreover, we observe that the filtration of the plasma inside the arterial wall is driven by a decreasing pressure gradient from the inner layer of the artery $\left(\Gamma_{\text {lumen }}\right)$ to the outer layer of the artery $\left(\Gamma_{\text {adv }}\right)$. By consequence we require that $p_{V}=p_{\text {lumen }}$ on $\Gamma_{\text {lumen }}$ and $p_{V}=p_{\text {adv }}$ on $\Gamma_{\text {adv }}$. We notice that $p_{\text {lumen }}$ is assumed to be uniform and independent of space and time variables on $\Gamma_{\text {lumen }}$. The velocity and the pressure in the arterial wall satisfy then the following equations

$$
\begin{cases}u_{V}=-\frac{k_{V}}{\mu_{V}} \nabla p_{V} & \text { in } V, \\ \nabla \cdot u_{V}=0 & \text { in } V, \\ p_{V}=p_{\text {lumen }} & \text { on } \Gamma_{\text {lumen }}, \\ p_{V}=p_{a d v} & \text { on } \Gamma_{\text {adv }}, \\ u_{V} \cdot \eta_{V}=0 & \text { on } \Gamma_{\text {wall }},\end{cases}
$$

where the boundaries $\Gamma_{\text {lumen }}, \Gamma_{\text {wall }}$ and $\Gamma_{\text {adv }}$ are defined in Figure 2 .

To simplify the model, we assume that the permeability $k_{V}$ and the viscosity $\mu_{V}$ are constants.

\section{Viscoelastic effects}

Viscoelastic models have been widely used to characterize mechanistic properties of the vascular tissues due to its ability to tailor both the viscoelastic relaxation function and the nonlinear elastic stress-strain relation. Numerous viscoelastic models, derived under different experimental conditions, have been proposed in the literature $[12,22-24,34,35]$. In what follows, we present a linear model (Maxwell-Wiechert model, [5]). The multiple relaxation times used in this model are well adapted to predict viscoelastic behaviour in living tissues ([24]). We postpone for a later section some considerations on the use of a nonlinear model (Fung's model, [11]).

In the Maxwell-Wiechert model, the relation between the stress and the strain is given by the following convolution integral

$$
\sigma_{V}(t)=-\left(k_{r} \varepsilon_{V}(t)+\int_{0}^{t} K(t-s) \frac{d \varepsilon_{V}}{d s}(s) d s\right)+\sigma_{S}(t),
$$


where $\sigma_{V}$ represents the normal stress which is assumed to be the most relevant component of the stress in the arterial wall, $\varepsilon_{V}$ is the effective strain in the arterial wall caused by accumulation of drug molecules, $\kappa_{r}$ is the Young's modulus of the spring arm and the convolution memory kernel $K$ is defined by $K(t-s)=\sum_{i=1}^{n} \kappa_{i} e^{-\frac{t-s}{\tau_{i}}}$, where $\tau_{i}=\frac{\eta_{i}}{\kappa_{i}}, i=1, \ldots, n$.

In (14), $\sigma_{S}(t)$ is the stress resulting from the deformation of the vessel caused by the stent. The constants $\kappa_{i}, i=1, \ldots, n$, represent the Young's modulus of the Maxwell arms while $\eta_{i}, i=$ $1, \ldots, n$, are their viscosities. This means that for $t=0$ the Young's modulus is $\kappa_{r}+\sum_{i=1}^{n} \kappa_{i}$ while for $t \rightarrow \infty$ its value is $\kappa_{r}$. We assume that $\sigma_{S}(t)$ does not depend on $x$ and $y$. This assumption is realistic for a homogeneous vessel wall and a circular cross section.

It should be noted that the negative sign in (14) indicates that $\sigma$ and $\varepsilon$ are of opposite sign. This represents the fact that the vessel wall acts like a barrier to the entry of the drug ([8]).

In [7] the authors established a non-linear relation between the strain $\epsilon$ and a concentration $C$, i.e. $\epsilon=\frac{C}{\rho-C}$ where $\rho$ stands for the density. To simplify the model we consider a linear approximation $\varepsilon_{V}(t)=\alpha_{m} C_{m, V}(t)+\epsilon_{S}(t), m=1, \ldots, 5, m \neq 2$. With this assumption and integrating (14) by parts we have

$$
\sigma_{m, V}(t)=-\alpha_{m}\left(\left(\kappa_{r}+\sum_{i=1}^{n} \kappa_{i}\right) C_{m, V}(t)-\sum_{i=1}^{n} \frac{\kappa_{i}}{\tau_{i}} \int_{0}^{t} e^{-\frac{t-s}{\tau_{i}}} C_{m, V}(s) d s\right)+\sigma_{S}(t)
$$

for $m=1, \ldots, 5, m \neq 2$.

Particular attention will be devoted to the case $n=1$ that is a mechanical analog composed by an elastic arm and a Maxwell arm. If we consider $K(t-s)=\left(\frac{\tau_{\sigma}}{\tau_{1}}-1\right) \kappa_{r} e^{-\frac{t-s}{\tau_{1}}}$ for $\tau_{1}=\frac{\eta_{1}}{\kappa_{1}}$ and $\tau_{\sigma}=\eta_{1} \frac{\kappa_{1}+\kappa_{r}}{\kappa_{1} \kappa_{r}}$, we obtain the so called 3 -parameter solid model which can also be deduced from the following differential formulation

$$
\sigma_{V}+\tau_{1} \frac{\partial \sigma_{V}}{\partial t}=-\kappa_{r}\left(\varepsilon_{V}+\tau_{\sigma} \frac{\partial \varepsilon_{V}}{\partial t}\right) .
$$

Equation (16) defines one of the simplest linear viscoelastic models that simultaneously captures the effects of creep and stress relaxation.

Equation (16) leads to the following formulation

$$
\sigma_{m, V}(t)=-\alpha_{m}\left(\kappa_{r} \frac{\tau_{\sigma}}{\tau_{1}} C_{m, V}(t)-\frac{\kappa_{1}}{\tau_{1}} \int_{0}^{t} e^{-\frac{t-s}{\tau_{1}}} C_{m, V}(s) d s\right)+\sigma_{S}(t)
$$

for $m=1, \ldots, 5, m \neq 2$.

\subsection{The complete model in the arterial wall}

The transport process that occurs in the vessel wall is due to convective transport, non-Fickian diffusion driven by the stress and reactions. It is described by the following set of equations $\frac{\partial C_{m, V}}{\partial t}=\nabla \cdot\left(\bar{D}_{m, V} \nabla C_{m, V}-u_{V} C_{m, V}\right)+\nabla \cdot\left(D_{\sigma} \nabla \sigma_{m, V}\right)+F_{m, V}\left(\mathcal{C}_{V}\right)$ in $V \times(0, T], m=1, \ldots, 5, m \neq 2$, 
where the stress $\sigma_{m, V}, m=1, \ldots, 5, m \neq 2$, is given by (15). In (18), $D_{\sigma}$ represents the "weight" of the non-Fickian diffusion and its physical meaning can be found in [7]. The idea is that the stress response to the strain represents an opposition to the motion of molecules, with concentrations $C_{m, V}, m=1, \ldots, 5, m \neq 2$. Assuming that convective field with speed $\nu_{V}$ is induced by the stress, we write

$$
-D_{\sigma} \nabla \sigma_{V}=\nu_{V} C_{V},
$$

where $C_{V}$ represents the total concentration of species. Identifying the stress with a pressure, we have from Darcy's law

$$
\nu_{V}=-\frac{\kappa_{V}}{\mu_{V}} \nabla \sigma_{V}
$$

where $\kappa_{V}$ is the permeability and $\mu_{V}$ is the viscosity of the fluid. From (19) and (20) we have

$$
D_{\sigma}=\frac{\kappa_{V}}{\mu_{V}} C_{V}
$$

In our model, $D_{\sigma}$ is assumed constant.

In what follows, particular attention will be paid to system (18) when the viscoelastic behaviour of the vessel wall is described by the 3-parameter solid model (17). In this case system (18) takes the form

$$
\frac{\partial C_{m, V}}{\partial t}=\nabla \cdot\left(D_{m, V} \nabla C_{m, V}-u_{V} C_{m, V}\right)+F_{m, V}\left(\mathcal{C}_{V}\right)+\int_{0}^{t} e^{-\frac{t-s}{\tau_{1}}} \nabla \cdot\left(D_{m, \sigma} \nabla C_{m, V}(s)\right) d s,
$$

in $V \times(0, T], m=1, \ldots, 5, m \neq 2$, where

$$
D_{m, V}=\bar{D}_{m, V}-\alpha_{m}\left(\kappa_{r}+\kappa_{1}\right) D_{\sigma}
$$

and $D_{m, \sigma}=\alpha_{m} \frac{\kappa_{1}}{\tau_{1}} D_{\sigma}$ for $m=1, \ldots, 5, m \neq 2$. We observe that when we replace the stress given by (17) in equation (18), the stress $\sigma_{S}(t)$, due to the deformation caused by the stent, disappears as its space derivatives are null.

To ensure the positivity of the Fickian diffusion coefficient $D_{m, V}$, we impose

$$
D_{\sigma}<\frac{\bar{D}_{m, V}}{\alpha_{m}\left(\kappa_{r}+\kappa_{1}\right)}
$$

This assumption guarantees that Fickian diffusion dominates the viscoelastic opposition, which is a physical condition for the effective penetration of drug in the vessel wall.

We note that the binding of drug molecules to specific sites in the vessel wall has not been considered in the present work. We refer to papers $[4,10,15,21]$ and [39] where different models account for the influence of binding in drug distribution.

The initial concentrations in the vessel wall are

$$
\left\{\begin{array}{l}
C_{1, V}(0)=1, \\
C_{m, V}(0)=0, \quad m=3,4,5 .
\end{array}\right.
$$


We represent by $J_{m, V}$ the mass flux in the vessel wall defined by

$$
J_{m, V}=-D_{m, V} \nabla C_{m, V}+u_{V} C_{m, V}-D_{m, \sigma} \int_{0}^{t} e^{-\frac{t-s}{\tau_{1}}} \nabla C_{m, V}(s) d s \quad \text { in } V \times \mathbb{R}^{+}, \underset{\substack{m=1, \ldots, 5, m \neq 2}}{ }
$$

Symmetry on $\Gamma_{\text {wall }}$ implies a non-flux condition,

$$
J_{m, V} \cdot \eta_{V}=0, \quad m=1, \ldots, 5, m \neq 2 .
$$

We also assume that adventitia is impermeable to all species present in the arterial wall. So the boundary condition (27) also holds for $\Gamma_{\mathrm{adv}}$. Since the drug, the oligomers and the lactic acid go directly from the arterial wall to the blood and are transported very fast away from the region of interest, we consider $J_{m, V} \cdot \eta_{V}=-\gamma_{m, V} C_{m, V}, m=3,4,5$, for the lumen boundary $\Gamma_{\text {lumen }}$, with an high transference rate $\gamma_{m, V}$. As the plasma penetrates from the blood artery into the arterial wall, we may consider a natural boundary condition $J_{1, V} \cdot \eta_{V}=\gamma_{1, V}\left(1-C_{1, V}\right)$ for the plasma.

\subsection{Interface coating-vessel wall boundary conditions}

To couple convection terms (4) and (13), on the interface boundary $\Gamma_{\text {coat }}$, the following matching conditions are applied

$$
\begin{cases}p_{S}=p_{V} & \text { on } \Gamma_{\text {coat }} \\ u_{S} \cdot \eta_{S}=-u_{V} \cdot \eta_{V} & \text { on } \Gamma_{\text {coat }}\end{cases}
$$

The interface conditions for concentration on the interface boundary $\Gamma_{\text {coat }}$ are described by

$$
\left\{\begin{array}{l}
C_{m, S}=C_{m, V} \\
J_{m, S} \cdot \eta_{S}=-J_{m, V} \cdot \eta_{V}
\end{array}\right.
$$

for $m=1, \ldots, 5, m \neq 2$. The first condition in (29) represents the continuity of the concentration while the second condition is the continuity of local fluxes. We recall that the subscript $m=2$ refers to PLA. In equation (29), the interface conditions do not apply to PLA. In fact PLA has a large molecular weight $\left(M_{W} \geq 1.2 \times 10^{5} \mathrm{~g} / \mathrm{mol}\right)$ compared to the other molecules present in the process and consequently it will not cross $\Gamma_{\text {coat }}$ (see [29]). As a result, $\Gamma_{\text {coat }}$ is impermeable to PLA and we have $J_{2, S} \cdot \eta_{S}=0$.

A more realistic interface condition considers that the coated stent, loaded with the drug, is covered by a second thin layer, called topcoat. This layer acts like a membrane between $S$ and $V$ to slow down the release rate from the stent into the arterial wall. The corresponding equations describe the flux of a chemical species across a membrane with respect to its concentration on both sides of the membrane. We postpone its mathematical description to a later section. 


\subsection{The coupled reaction-diffusion-convection system}

In summary, the coupled system of reaction-diffusion-convection equations in the coating stent and the wall reads as follows:

$$
\left\{\begin{aligned}
\frac{\partial C_{m, S}}{\partial t} & =\nabla \cdot\left(D_{m, S} \nabla C_{m, S}-u_{S} C_{m, S}\right)+F_{m, S}\left(\mathcal{C}_{S}\right) & & \text { in } S \times \mathbb{R}^{+}, m=1, \ldots, 5, \\
\frac{\partial C_{m, V}}{\partial t} & =\nabla \cdot\left(D_{m, V} \nabla C_{m, V}-u_{V} C_{m, V}\right)+F_{m, V}\left(\mathcal{C}_{V}\right) & & \\
& +\int_{0}^{t} e^{-\frac{t-s}{\tau_{1}}} \nabla \cdot\left(D_{m, \sigma} \nabla C_{m, V}(s)\right) d s & & \text { in } V \times \mathbb{R}^{+}, m=1, \ldots, 5, m \neq 2,
\end{aligned}\right.
$$

where $u_{S}$ and $u_{V}$ are solutions of (4) and (13) respectively. The initial conditions in the stent coating and arterial wall are defined by (7) and (25) respectively and the boundary and interface conditions are summarized by

$$
\begin{cases}J_{m, S} \cdot \eta_{S}=0 & \text { on } \Gamma_{\text {strut }}, m=1, \ldots, 5, \\ J_{2, S} \cdot \eta_{S}=0 & \text { on } \Gamma_{\text {coat }}, \\ C_{m, S}=C_{m, V} & \text { on } \Gamma_{\text {coat }}, m=1, \ldots, 5, m \neq 2, \\ J_{m, S} \cdot \eta_{S}=-J_{m, V} \cdot \eta_{V} & \text { on } \Gamma_{\text {coat }}, m=1, \ldots, 5, m \neq 2, \\ J_{1, V} \cdot \eta_{V}=\gamma_{1, V}\left(1-C_{1, V}\right) & \text { on } \Gamma_{\text {lumen }}, \\ J_{m, V} \cdot \eta_{V}=-\gamma_{m, V} C_{m, V} & \text { on } \Gamma_{\text {lumen }}, m=3,4,5, \\ J_{m, V} \cdot \eta_{V}=0 & \text { on } \Gamma_{\text {wall }} \cup \Gamma_{\text {adv }}, m=1, \ldots, 5, m \neq 2 .\end{cases}
$$

\section{$3 \quad$ Some analytical results}

In what follows we analyse the behaviour of the total mass of species $M(t)$ in the model. We define

$$
\mathcal{M}(t)=\int_{S} \mathcal{C}_{S} d S+\int_{V} \mathcal{C}_{V} d V
$$

where $\int_{S} \mathcal{C}_{S} d S=\sum_{m=1}^{5} \int_{S} C_{m, S} d S$ and $\int_{V} \mathcal{C}_{V} d V=\sum_{\substack{m=1 \\ m \neq 2}}^{5} \int_{V} C_{m, V} d V$.

Using (30), we obtain

$$
\begin{aligned}
\mathcal{M}^{\prime}(t) & =\sum_{\substack{m=1 \\
S}}^{5} \nabla \cdot\left(D_{m, S} \nabla C_{m, S}-u_{S} C_{m, S}\right) d S+\sum_{m=1}^{5} \int_{S} F_{m, S}\left(\mathcal{C}_{S}\right) d S \\
& +\sum_{\substack{m=1 \\
m \neq 2}}^{5} \int_{V} \nabla \cdot\left(D_{m, V} \nabla C_{m, V}-u_{V} C_{m, V}\right) d V+\sum_{\substack{m=1 \\
m \neq 2}} \int_{V} F_{m, V}\left(\mathcal{C}_{V}\right) d V \\
& +\sum_{\substack{m=1 \\
m \neq 2}}^{5} \int_{V} \int_{0}^{t} e^{-\frac{t-s}{\tau_{1}}} \nabla \cdot\left(D_{m, \sigma} \nabla C_{m, V}(s)\right) d s d V .
\end{aligned}
$$


Integrating over space and applying external boundary conditions, we have

$$
\begin{aligned}
\mathcal{M}^{\prime}(t) & =\sum_{\substack{m=1 \\
m \neq 2}}^{5} \int_{\Gamma_{\text {coat }}} J_{m, S} \cdot \eta_{S} d s+\sum_{\substack{m=1 \\
m \neq 2}}^{5} \int_{\Gamma_{\text {coat }}} J_{m, V} \cdot \eta_{V} d s+\int_{S} \sum_{m=1}^{5} F_{m, S}\left(\mathcal{C}_{S}\right) d S+\int_{V} \sum_{\substack{m=1 \\
m \neq 2}}^{5} F_{m, V}\left(\mathcal{C}_{V}\right) d V \\
& +\gamma_{1, V} \int_{\Gamma_{\text {lumen }}}\left(1-C_{1, V}\right) d s-\sum_{m=3}^{5} \gamma_{m, V} \int_{\Gamma_{\text {lumen }}} C_{m, V} d s .
\end{aligned}
$$

Let

$$
\begin{aligned}
& \Delta M_{\Gamma_{\text {lumen }}}(t)=\sum_{\substack{m=1 \\
m \neq 2}}^{5} \gamma_{m, V} \int_{\Gamma_{\text {lumen }}} C_{m, V}(t) d s \\
& \Delta M_{H}(t)=\int_{S} \kappa_{2, S} C_{1, S}(t) C_{3, S}(t)\left(1+\beta C_{4, S}(t)\right) d S+\int_{V} \kappa_{1, V} C_{1, V}(t) C_{3, V}(t)\left(1+\gamma C_{4, V}(t)\right) d V .
\end{aligned}
$$

We note that $\Delta M_{\Gamma_{\text {lumen }}}(t)$ represents the mass per unit time of molecules (except PLA) that enters in $\Gamma_{\text {lumen }}$ at the instant $t$ while $\Delta M_{H}(t)$ stands for the total mass of hydrolyzed oligomers that enter per unit time in the stent and the vessel wall at the same instant.

Using interface condition on $\Gamma_{\text {coat }}$ we easily establish

$$
\mathcal{M}(t)=\mathcal{M}(0)+\gamma_{1, V}\left|\Gamma_{\text {lumen }}\right| t-\int_{0}^{t} \Delta M_{H}(\mu) d \mu-\int_{0}^{t} \Delta M_{\Gamma_{\text {lumen }}}(\mu) d \mu .
$$

This last equation means that the total mass in the system at a certain time $t, t \in[0, T]$, is given by the difference between the initial mass added with the mass of plasma that enters in the system until time $t$ and the cumulative masses of molecules in $\Gamma_{\text {lumen }}$, the stent and the vessel wall.

A stability analysis of the model is presented in Annex $A$.

\section{$4 \quad$ Numerical results}

We define a variational formulation of the complete model (25) - (31). Based on this formulation a $P_{1}$ finite element method is proposed (eq. (57) in Annex B).

All experiments have been carried out with the open source PDE solver freeFEM ++ considering the triangulation plotted in Figure 4, with 14741 elements (7623 vertices) for the vessel wall and 393 elements (264 vertices) for each stent. The time integration of equation (57) has been performed using an implicit-explicit backward formula in the time grid $\left\{t_{n} ; n=0,1, \ldots, N\right\}, t_{0}=0, t_{N}=T$ and with time step size $\Delta t=10^{-3}$.

An implicit-explicit (IMEX) method is defined by integrating (57) with an implicit Euler method where the diffusion and the convective terms are considered implicitly with explicit diffusion coefficients. In the discretization of the reaction terms, we adopt an IMEX approach which converts each nonlinear reaction into a linear one at each time level: for example, in the equation of the transport of plasma in the coating at time level $t_{n+1}$, we use the value of $C_{1, S, h}$ (the discrete approximation of $\left.C_{1, S}\right)$ at time level $t_{n+1}$ while we use concentrations $C_{m, S, h}, m=2, \ldots, 5$, at time level $t_{n}$.

To compute the finite element solutions we need to evaluate some convolution integrals. To avoid 
the use of quadrature rules, we generate and implicitly solve a set of ordinary differential equations whose solutions are those integrals.

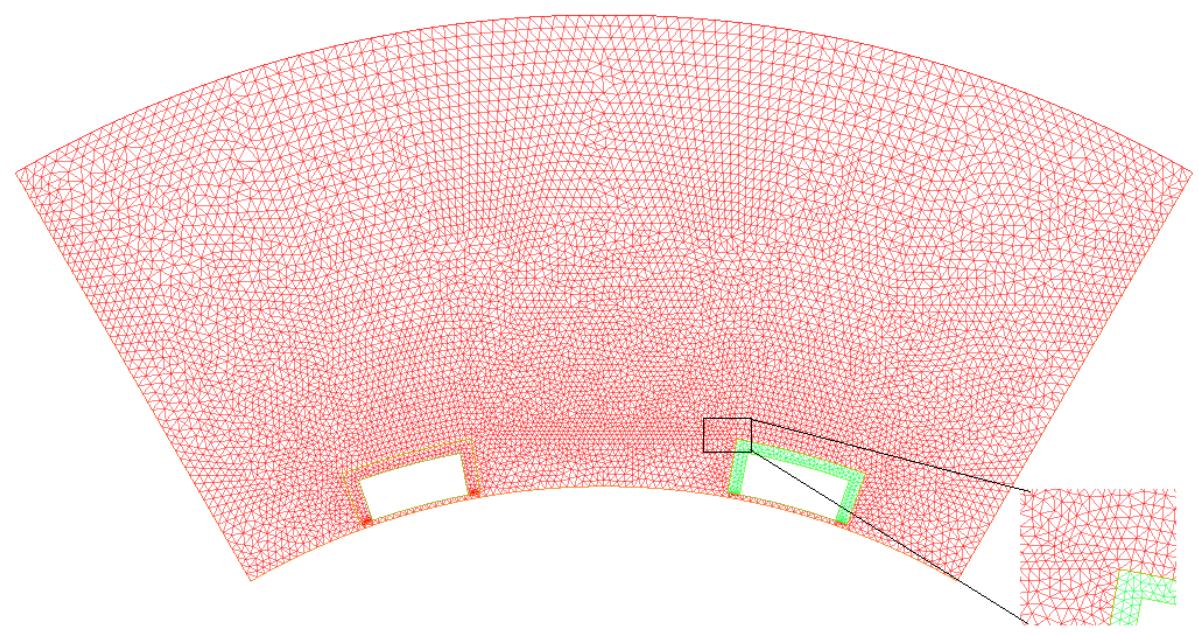

Figure 4: Triangulations in the stent and in the arterial wall.

The interested reader is referred to annexes $A$ and $B$ for mathematical details.

We define the mass in the coated stent and in the arterial wall respectively by

$$
\begin{aligned}
& \mathcal{M}_{m, S, h}\left(t_{n}\right)=\int_{S_{h}} C_{m, S, h}\left(t_{n}\right) d S, m=1, \ldots, 5, \\
& \mathcal{M}_{m, V, h}\left(t_{n}\right)=\int_{V_{h}} C_{m, V, h}\left(t_{n}\right) d V, m=1, \ldots, 5, m \neq 2,
\end{aligned}
$$

where $\mathcal{M}_{m, j, h}\left(t_{n}\right), j=S, V$, are the numerical approximations for masses at time level $t_{n}$.

There is a large degree of variability in most parameters exhibited in the literature, due to the particular approach used in their estimation (as for example in vivo vs. in vitro, human vs. animal). In this paper we have extracted the values of the parameters from [28, 29], and [41]. Their physical meaning and values have been displayed in Table 1 . The thickness of media $\left(2 \times 10^{-2} \mathrm{~cm}\right)$ and stent coating $\left(5 \times 10^{-4} \mathrm{~cm}\right)$ have been extracted from $[28,41]$. 


\begin{tabular}{|c|c|c|}
\hline Parameter/Variable & Definition & Value \\
\hline & Stent coating & \\
\hline$\overline{D_{1, S}^{0}}$ & diffusion coefficient of plasma & $10^{-8} \mathrm{~cm}^{2} / \mathrm{s}$ \\
\hline$D_{2, S}^{0, N}$ & diffusion coefficient of PLA & $10^{-15} \mathrm{~cm}^{2} / \mathrm{s}$ \\
\hline$D_{3, S}^{0, D}$ & diffusion coefficient of oligomers & $10^{-10} \mathrm{~cm}^{2} / \mathrm{s}$ \\
\hline$D_{4, S}^{0}$ & diffusion coefficient of lactic acid & $2 \times 10^{-10} \mathrm{~cm}^{2} / \mathrm{s}$ \\
\hline$D_{5, S}^{0, N}$ & diffusion coefficient of drug & $10^{-8} \mathrm{~cm}^{2} / \mathrm{s}$ \\
\hline$\kappa_{1, S}$ & rate of first reaction & $10^{-6} \mathrm{~cm}^{2} / \mathrm{g} . \mathrm{s}$ \\
\hline$\kappa_{2, S}$ & rate of second reaction & $10^{-7} \mathrm{~cm}^{2} / \mathrm{g} . \mathrm{s}$ \\
\hline$k_{S}$ & permeability of fluid & $2 \times 10^{-14} \mathrm{~cm}^{2}$ \\
\hline$\mu_{S}$ & viscosity of fluid & $7.2 \times 10^{-2} \mathrm{~g} / \mathrm{cm} . \mathrm{s}$ \\
\hline$\alpha$ & dimensional parameter & $1 \mathrm{~s} / \mathrm{cm}^{2}$ \\
\hline$\beta$ & dimensional parameter & $10 \mathrm{~s} / \mathrm{cm}^{2}$ \\
\hline & Arterial wall & \\
\hline$\overline{D_{1, V}}$ & diffusion coefficient of plasma & $10^{-8} \mathrm{~cm}^{2} / \mathrm{s}$ \\
\hline $\bar{D}_{3, V}$ & diffusion coefficient of oligomers & $10^{-10} \mathrm{~cm}^{2} / \mathrm{s}$ \\
\hline $\bar{D}_{4, V}$ & diffusion coefficient of lactic acid & $2 \times 10^{-10} \mathrm{~cm}^{2} / \mathrm{s}$ \\
\hline $\bar{D}_{5, V}$ & diffusion coefficient of drug & $10^{-10} \mathrm{~cm}^{2} / \mathrm{s}$ \\
\hline$D_{\sigma}$ & viscoelastic diffusion coefficient & $5 \times 10^{-8} \mathrm{~g} /(\mathrm{cmsPa})$ \\
\hline$\tau_{1}$ & relaxation time & $0.5 s$ \\
\hline$\kappa_{r}$ & Young modulus & $1.2 M P a$ \\
\hline$\kappa_{1}$ & Young modulus of the arm & $1 M P a$ \\
\hline$\kappa_{1, V}$ & rate of first reaction & $10^{-6} \mathrm{~cm}^{2} / \mathrm{g} . \mathrm{s}$ \\
\hline$k_{V}$ & permeability of fluid & $10^{-15} \mathrm{~cm}^{2}$ \\
\hline$\mu_{V}$ & viscosity of fluid & $5 \times 10^{-2} \mathrm{~g} / \mathrm{cm} . \mathrm{s}$ \\
\hline$\gamma$ & dimensional parameter & $10 \mathrm{~s} / \mathrm{cm}^{2}$ \\
\hline$p_{\text {lumen }}$ & pressure on lumen & $10^{2} \mathrm{mmHg}$ \\
\hline$p_{\text {adv }}$ & pressure on adventitia & $0 \mathrm{mmHg}$ \\
\hline
\end{tabular}

Table 1: Values for the parameters and variables in the stent coating and in the arterial wall.

We note that the arterial wall is anisotropic and consequently diffusion has different values in circumferential and axial directions. To simplify our model we use a mean value for the diffusion coefficient. A more realistic description will be used in a future work.

The values used in this study correspond to the drug paclitaxel applied in Taxus paclitaxel eluting stent from Boston Scientific, Natick, MA, USA. It is a hydrophobic therapeutic agent to control migration of smooth muscle cells from endothelium caused by in-stent restenosis. Other types of drug such as sirolimus, also known as rapamycin, $\left(D_{5, S}=1 \times 10^{-9},[18]\right)$ or heparin $\left(D_{5, S}=\right.$ $\left.7.7 \times 10^{-8},[41]\right)$ are used in some commercial DES. 


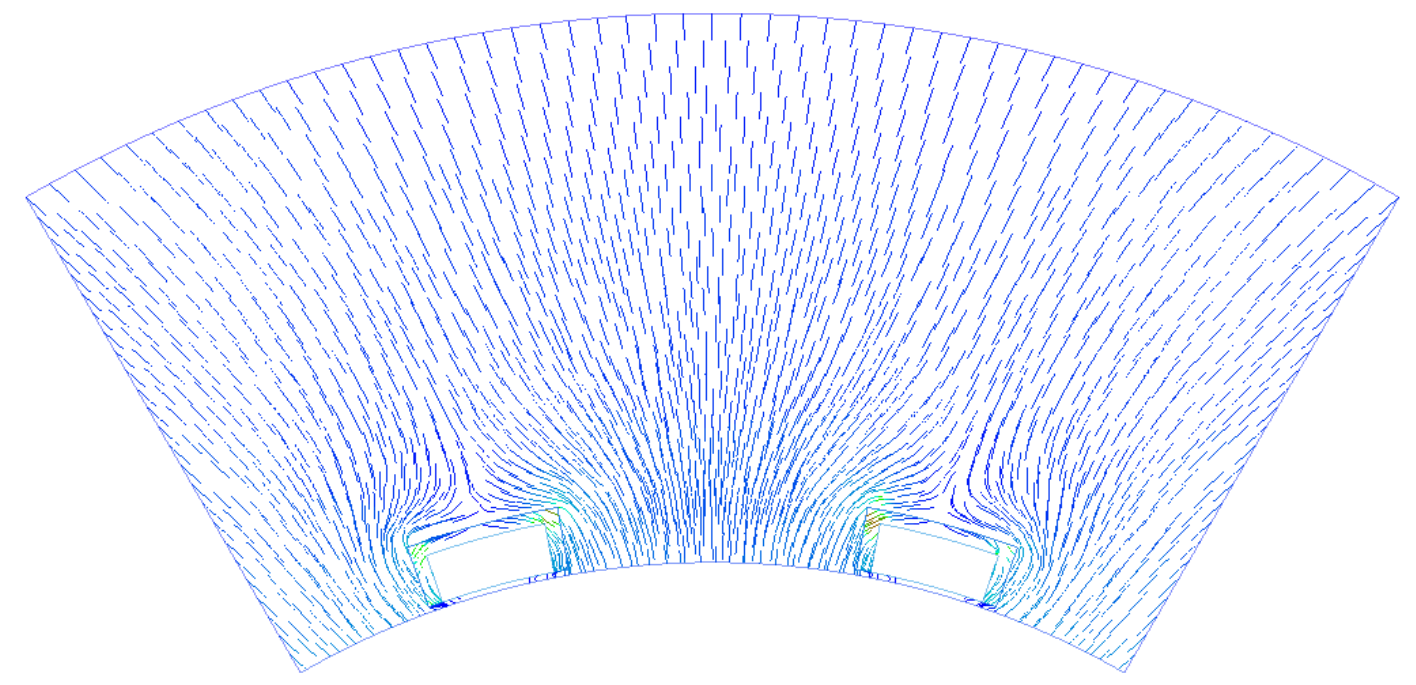

(a) Velocity field

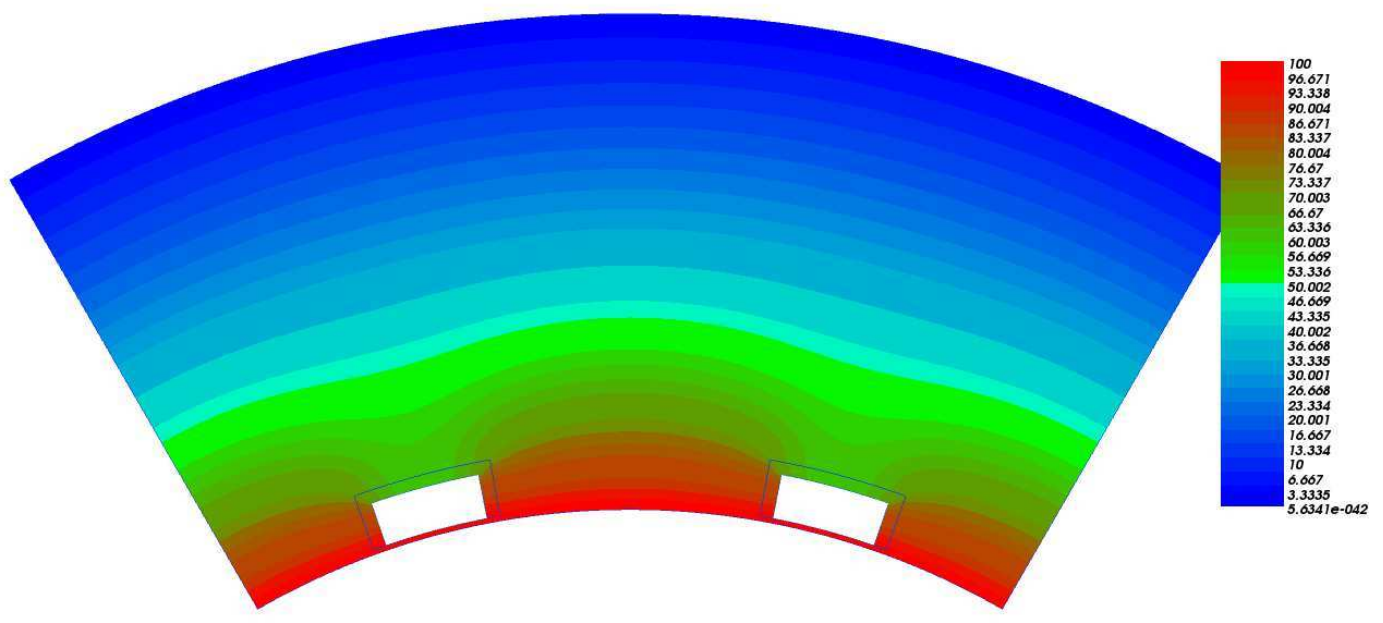

(b) Pressure distribution

Figure 5: Velocity field and pressure distribution in the coupled system.

We impose a pressure difference between the inner surface $\left(\Gamma_{\text {lumen }}\right)$ and the outer surface $\left(\Gamma_{\text {adv }}\right)$ of the arterial wall. A velocity field in the coupled stent-wall system is caused by this pressure jump (Figure $5(a)$ ). The average magnitude of the velocity in the stented arterial wall observed is $10^{-6} \mathrm{~cm} / \mathrm{s}$ which agrees with the value presented in [41].

The pressure drop given by system (40) is shown in Figure 5. While pressure on the interface boundary $\Gamma_{\text {coat }}$ is around $76.88 \mathrm{mmHg}$, it is observed that the average pressure in the arterial wall and in the stent are $35.93 \mathrm{mmHg}$ and $75.34 \mathrm{mmHg}$ respectively.

The release of the drug from the stent into the vessel wall is illustrated in Figure 6. As time evolves the mass of the drug increases in the arterial wall. 


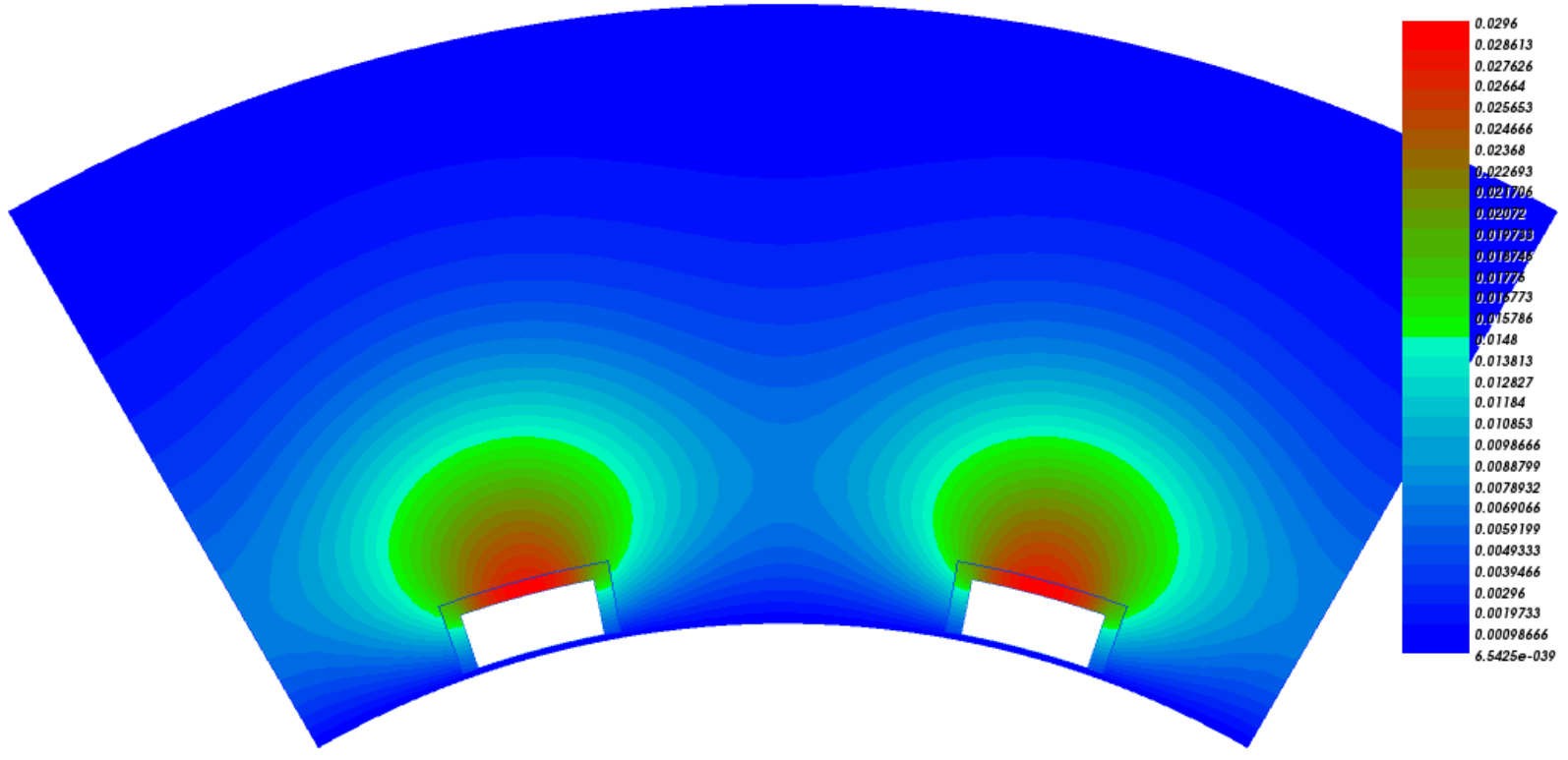

(a) 1 month

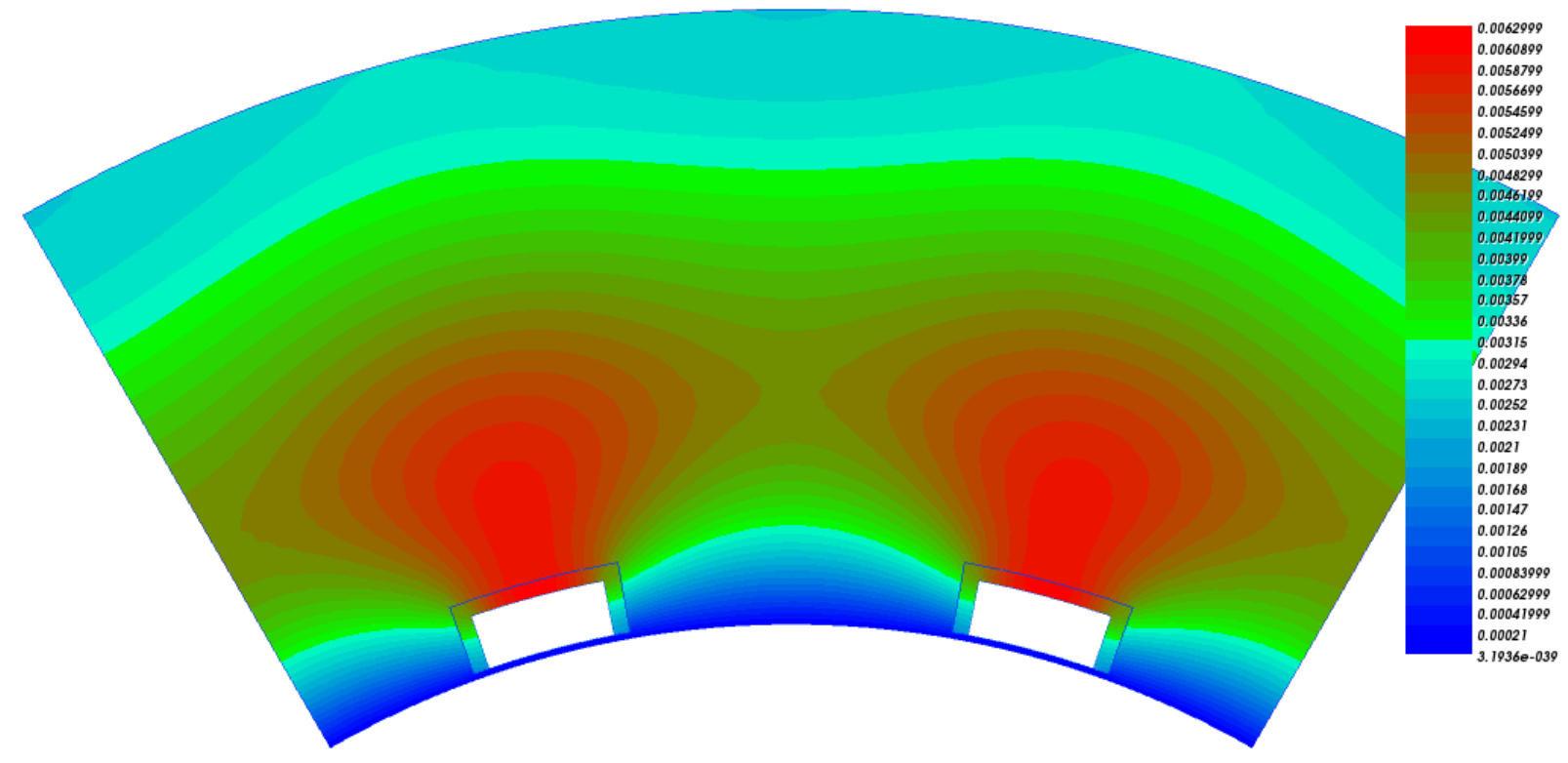

(b) 3 months

Figure 6: Evolution of concentration in the stent and in the vessel wall.

The behavior of the mass of drug, the mass of PLA and the amount of fluid in the biodegradable stent is shown in Figure 7. The drug presents a steep initial gradient and gradually vanishes after 
three months. The penetration of the fluid in the stent presents a steep initial slope and after around 20 days achieves a steady state. We can also observe in Figure 7 that as PLA degrades, the release rate of drug decreases.

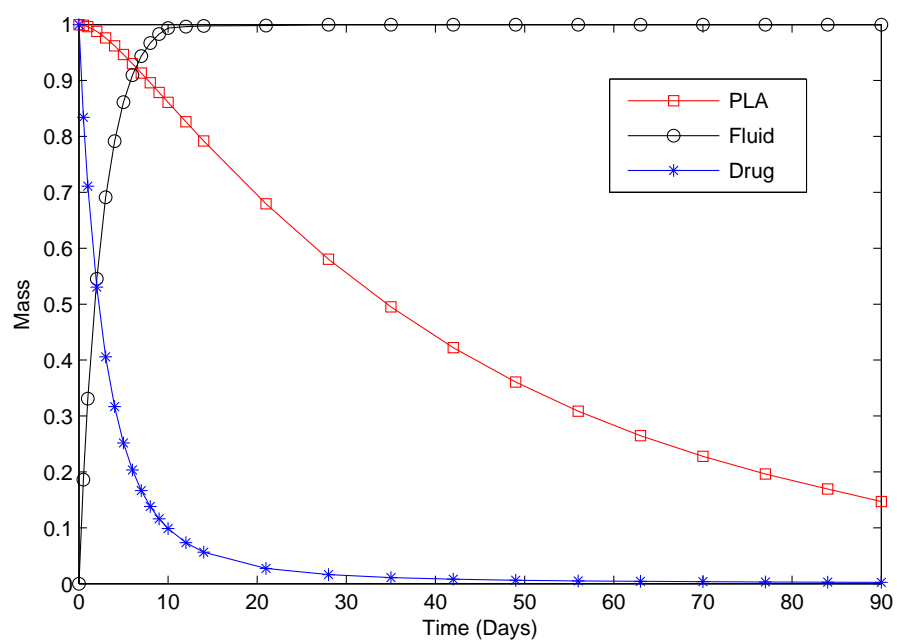

Figure 7: Mass behaviour of fluid, PLA and drug in the stent during 90 days.

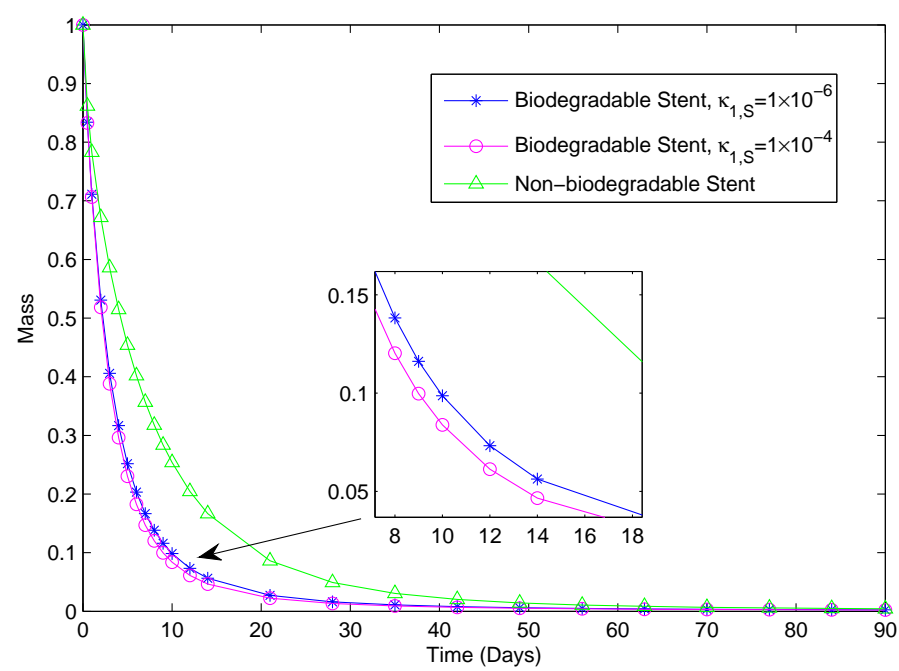

Figure 8: Mass of drug released from a biodegradable stent versus the mass released from a nonbiodegradable stent.

The release of drug from a biodegradable stent and a non-biodegradable stent are compared in Figure 8. We observe that due to the degradation of the polymer, the drug release from a biodegradable stent is faster than the drug release from a non-biodegradable stent. The drug release rate directly depends on the reaction rate $\kappa_{1, S}$.

The influence of the stiffness of the vessel wall in the diffusion process of the drug is shown in Figure 9. A healthy coronary artery with Young's modulus $\kappa_{r}=1.2 \mathrm{MPa}$ (see [13]) is compared with a highly diseased coronary artery with Young's modulus $\kappa_{r}=4.1 \mathrm{MPa}$ (see [25]). 
As $\kappa_{r}$ increases due to age or atherosclerosis, the vessel wall is less elastic, that is more stiff, and less drug penetrates the coronary wall for short times. This is an interesting result from the medical viewpoint, because cardiovascular morbidity is related with arterial stiffness [16]. The dependence of the drug concentration on the vessel stiffness suggests that DES can be tailored according to the severity of the arterial disease. In our numerical experiments the drug reduction of mass in the initial days of the process could be compensated by adding 2 percent drug to the initial concentration.

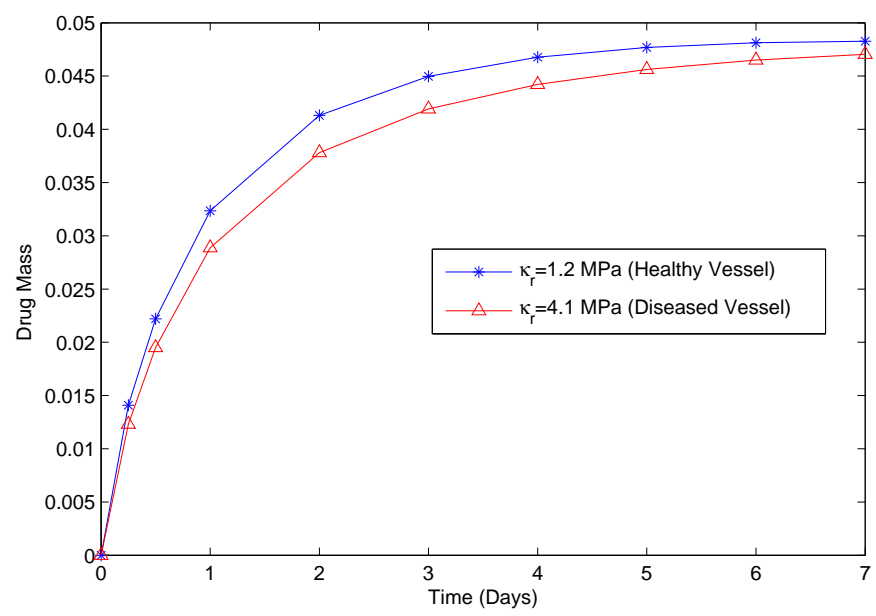

(a) short time

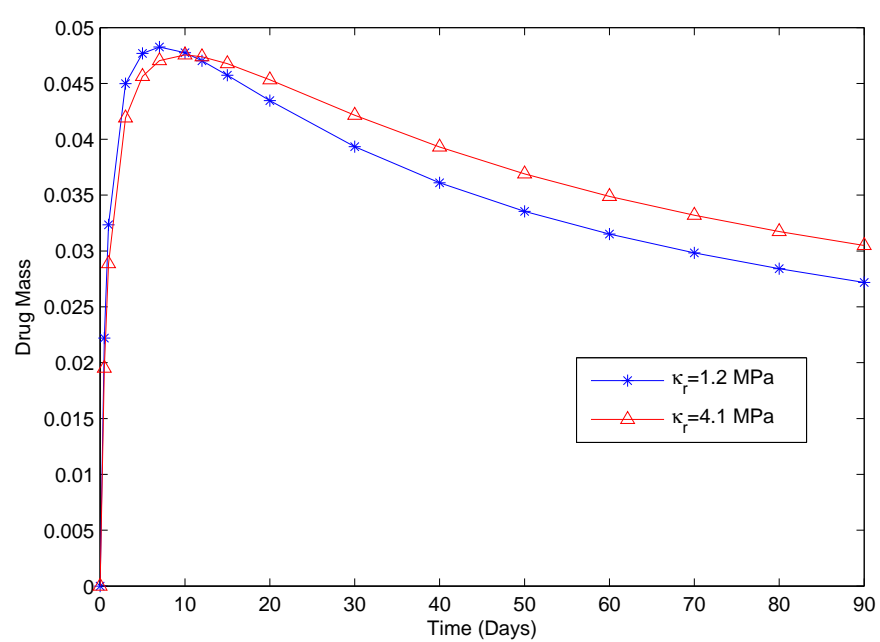

(b) long time

Figure 9: Effect of $\kappa_{r}$ on the mass of drug that enters in the vessel wall in short and long times.

The long term influence of stiffness of the coronary wall in the transport of the drug is shown in Figure $9(b)$. In the beginning of the treatment, a diseased coronary wall receives less drug due to its large $\kappa_{r}$ when we compare with a healthy coronary wall. A crossing occurs after the initial times around day 15. This finding is justified by the fact that the stiffness of the vessel wall imposes 
a resistance to the penetration of the drug in the beginning of the process and leads to a drug accumulation in the long time.

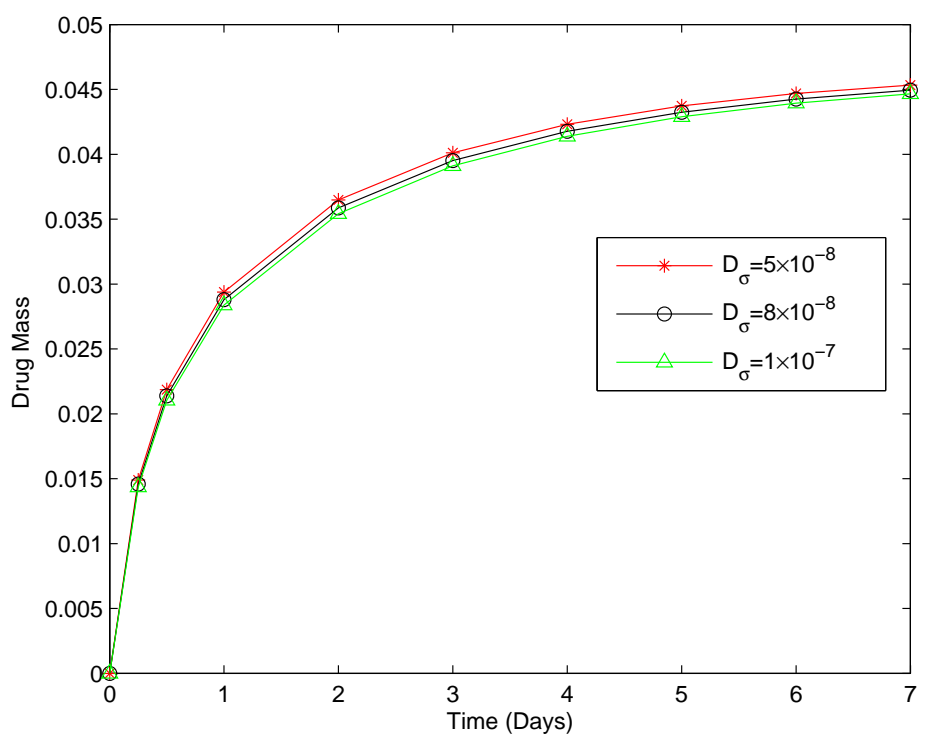

Figure 10: The effect of the viscoelastic diffusion coefficient $D_{\sigma}$ on the drug release in the arterial wall.

The effect of viscoelastic diffusion coefficient $D_{\sigma}$ on the drug release is shown in Figure 10. When $D_{\sigma}$ increases, we can expect less accumulation of drug in the vessel wall in the beginning of the process. This fact is just due to an increasing of the resistance of the vessel wall to the drug penetration. We note that for $D_{\sigma}=0$ we obtain a pure Fickian model. The values for $D_{\sigma}$ in Figure 10, satisfy relation (24).

When an additional thin layer named topcoat is applied to the PLA matrix instead of the interface conditions (29) we consider the following interface conditions

$$
\left\{\begin{array}{l}
J_{m, S} \cdot \eta_{S}=P_{c}\left(C_{m, S}-C_{m, V}\right), \\
J_{m, S} \cdot \eta_{S}=-J_{m, V} \cdot \eta_{V},
\end{array}\right.
$$

for $m=1, \ldots, 5, m \neq 2$, where $P_{c}$ is the permeability of the interface layer $\Gamma_{\text {coat }}$. The first condition in (35) is the second Kedem-Katchalsky equation (see [28] and the references therein). We remark that the topcoat is used to slow down the release rate of the drug. 


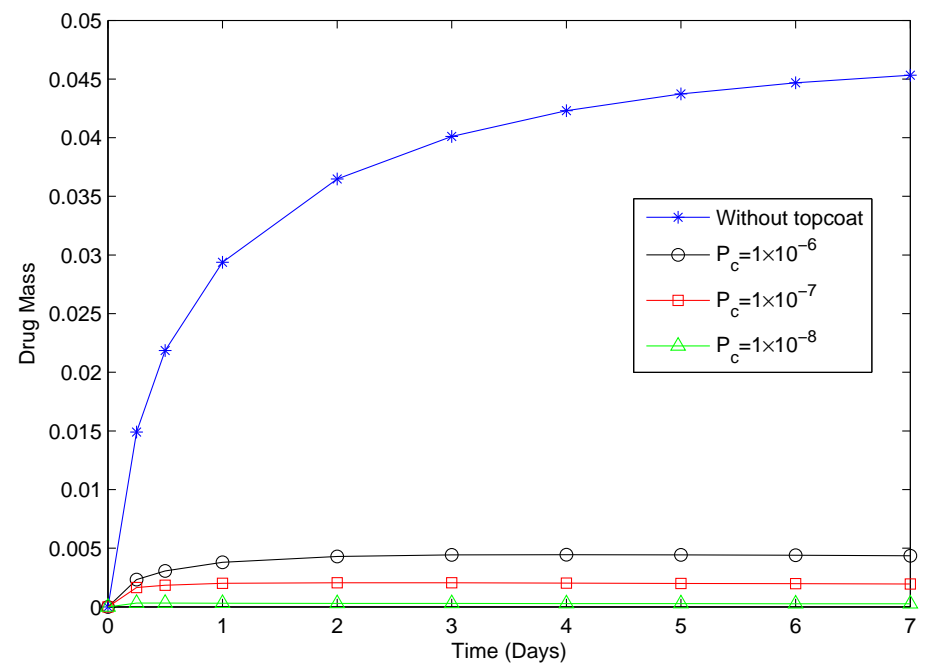

Figure 11: The effect of topcoat on the drug release in the vessel wall.

Figure 11 presents the effect of permeability of the interface layer $\Gamma_{\text {coat }}$ on the drug release when a topcoat is applied to the PLA. The accumulation of drug will decrease when a topcoat with smaller permeability is applied to the coated stent. This means that the release of drug from the stent into a vessel wall can be controlled by applying topcoats with different permeabilities.

\section{An alternative model to Maxwell-Wiechert model}

Fung's quasilinear viscoelastic model ([11]) is commonly used to describe the viscoelastic properties of the living tissues. Several authors consider that Fung's quasilinear viscoleastic model is a simple method to incorporate nonlinearity and viscoelasticity and is a good model for living tissues with moderate deformation $([1,11,24,38])$.

The aim of this subsection is to show that the effect of the rheological properties of the vessel wall, on drug permeation, are described analogously by Maxwell-Wiechert model and Fung's model.

In the framework of Fung's model, the relation between stress and strain is given by the following convolution integral

$$
\sigma_{V}(t)=-\int_{0}^{t} \tilde{K}(t-s) \frac{d \sigma^{e}\left(\varepsilon_{V}\right)}{d s}(s) d s
$$

where

$$
\tilde{K}(t-s)=\frac{1+c \int_{\tau_{1}}^{\tau_{2}} \frac{1}{\tau} e^{-\frac{t-s}{\tau}} d \tau}{1+c \ln \left(\frac{\tau_{2}}{\tau_{1}}\right)},
$$

and

$$
\sigma^{e}\left(\varepsilon_{V}(t)\right)=\lambda_{1}\left(e^{\lambda_{2} \varepsilon_{V}(t)}-1\right) \simeq \lambda_{1} \lambda_{2} \varepsilon_{V}(t)
$$




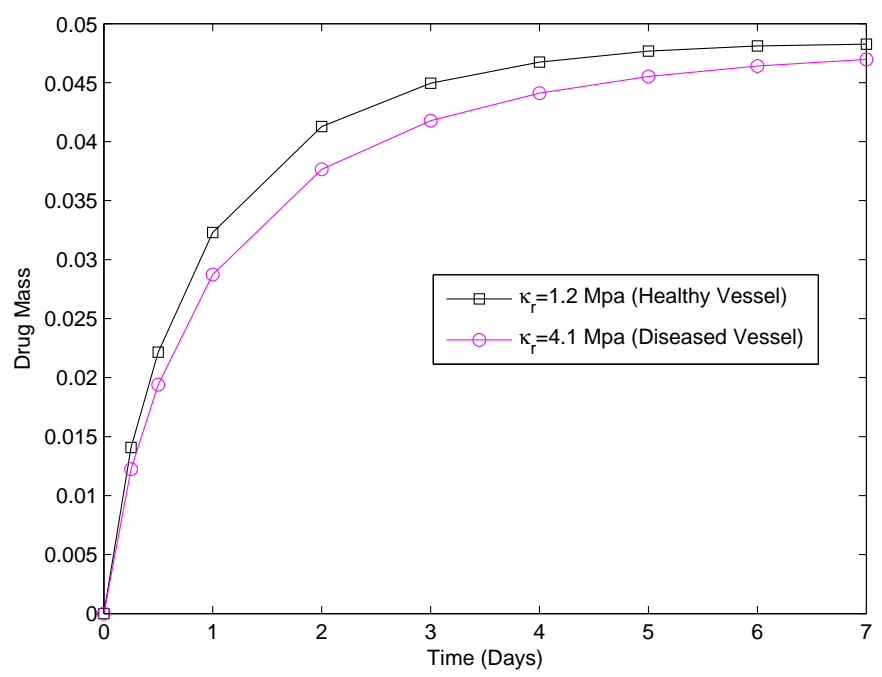

(a) short time

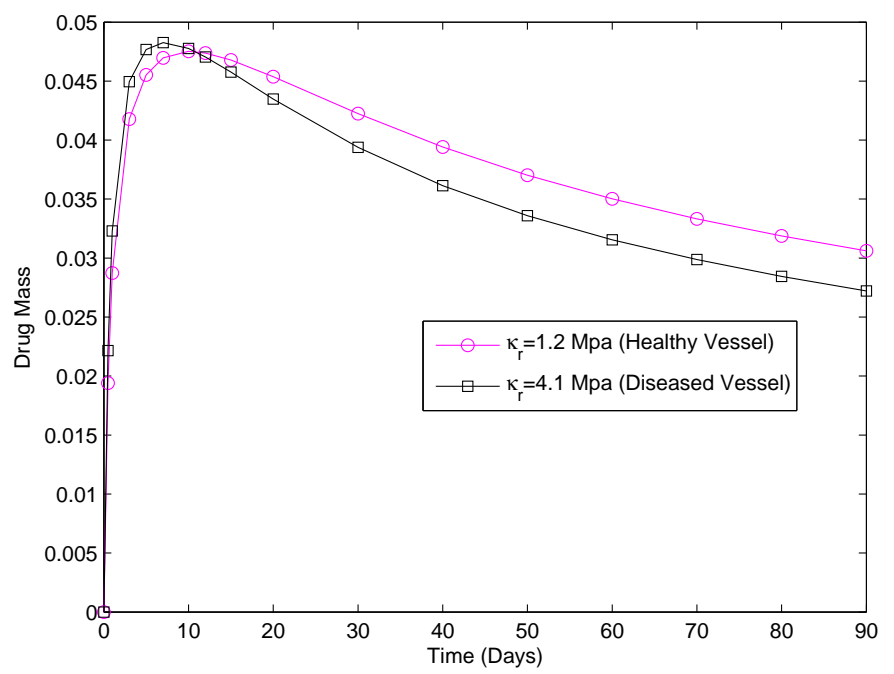

(b) long time

Figure 12: Effect of parameter $\tilde{\kappa}_{r}$ on the drug release in the vessel wall (Fung's model) in short and long times.

In (37), $c>0$ represents the influence of viscous effects, $\tau_{1}$ and $\tau_{2}$ represent the short-term and long-term time constants respectively. In $(38), \sigma^{e}\left(\varepsilon_{V}\right)$ represents the instantaneous nonlinear elastic strain, $\lambda_{1}>0$ is the elastic stress constant ( $\left.\mathrm{MPa}\right)$ and $\lambda_{2}$ is a non-dimensional parameter representing the nonlinearity of instantaneous elastic response.

Replacing (37) and (38) into (36), we obtain

$$
\sigma_{V}(t)=-\tilde{k}_{r}\left(\varepsilon_{V}(t)+c \int_{0}^{t} \int_{\tau_{1}}^{\tau_{2}} \frac{1}{\tau} e^{-\frac{t-s}{\tau}} d \tau \frac{d \varepsilon_{V}}{d s}(s) d s\right)+\sigma_{S}(t),
$$


where $\tilde{k}_{r}=\frac{\lambda_{1} \lambda_{2}}{1+c \ln \left(\frac{\tau_{2}}{\tau_{1}}\right)}$ and $\sigma_{S}(t)$ is as before the stress response to the stent deformation. The quasilinear viscoelastic model has five material parameters (three for the reduced relaxation function (Equation (37)) and two for the elastic response (Equation (38)) which must be determined experimentally. Although some estimations are available in the literature for ligaments ([17]), femur-MCL-tibia complexes ([1]) and spinal tissue ([38]), to the best knowledge of the authors, physiological values of these five parameters are not available in the case of coronary walls. Due to the lack of appropriate information, we fix four parameters $\lambda_{1}=0.2 \mathrm{Mpa}, \lambda_{2}=25$, $\tau_{1}=0.5 \mathrm{~s}$ and $\tau_{2}=1800 \mathrm{~s}$ and choose $c=0.37$ to have $\tilde{\kappa}_{r}=1.2 \mathrm{Mpa}([13])$ for healthy arterial wall and $c=0.02$ to have $\tilde{\kappa}_{r}=4.1 \mathrm{Mpa}([25])$ for a highly diseased arterial wall.

The plots in Figure 12 show that the profile of drug release exhibits the same qualitative behavior as before. The barrier to drug permeation of stiff vessel walls, in the first period of drug delivery, is a clinical finding suggested by Fung's and Maxwell-Wiechert mechanistic models.

\section{Conclusions}

In recent years mathematical modeling has become an effective tool to simulate drug delivery processes. In the case of drug eluting stents it leads to a deeper understanding of the drug release mechanisms in the biodegradable coating and in the vessel wall. Although the cardiovascular drug delivery depends on very complex biochemical and physiological phenomena, we believe that a simplified release model can help to provide understanding of the role of some of the key factors such as the stiffness of the arterial wall.

In this paper we present a coupled model to simulate drug delivery from a stent to a vessel wall. The coating of the stent is biodegradable and the viscoelastic properties of the vessel wall are included in the model. From the numerical viewpoint two particular aspects of clinical importance are addressed: the influence of the viscoelasticity of the vessel wall and the effect of the permeability of the stent coating.

Concerning the first aspect we show that during an initial period of time the permeation of drug in the vessel is affected by its stiffness: the total mass of drug that enters the vessel is a decreasing function of the Young's modulus. Patients who need a cardiovascular stent generally have atherosclerosis and consequently stiff vessels that have high Young's modulus. To prevent an inflammatory response and the smooth muscle cell growth a correct concentration of drug must penetrate the vessel from the moment when the stent is implanted. Interestingly such behavior changes for late times: the amount of drug in the vessel in an increasing function of the stiffness. The second aspect we want to stress is the control of the release profile according to the permeability of the coating: release can be speeded up or delayed as different polymers are used.

The results presented in the paper are physiologically and physically sound, which suggests its face validity. However, as written before, we made some mathematical assumptions that should be relaxed in order to obtain a more realistic model. We mention the circular cross section of the vessel, the homogeneity of the vessel wall and the complete embedding of the stent from its insertion. These aspects will be addressed in the near future by considering:

1. Real geometries obtained from OCT images. Deviations relatively to a circular cross section result in an initial stress (caused by the stent) dependent on space. 
2. Heterogeneity of the vessel wall using real images where lipidic and calcium plaques are identified. Diffusive and rheological properties of the vessel will depend on space.

3. The proliferation of neo-intima cells that produce the coverage of the stent and ultimately the restenosis of the vessel.

The inclusion of these aspects and the use of OCT data to validate the model will represent a step forward for the field.

\section{Acknowledgements}

This work was partially supported by the Centro de Matemática da Universidade de Coimbra (CMUC), funded by the European Regional Development Fund through the program COMPETE and by the Portuguese Government through the FCT under the project, PEst-C/MAT/UI0324/2013, by the project UTAustin/MAT/0066/2008 and also FCT-Grant SFRH/BD/51167/2010.

\section{A Weak formulation of the continuous model}

\section{A.1 Porous media problem}

In order to find the pressure drop in the stented vessel wall, as $k_{j}$ and $\mu_{j}, j=S, V$, are constants, it is convenient to rewrite the equations (4), (13) and (28) in terms of pressure drop in the following coupled form

$$
\begin{cases}-\nabla \cdot\left(\frac{k_{V}}{\mu_{V}} \nabla p_{V}\right)=0 & \text { in } V, \\ -\nabla \cdot\left(\frac{k_{S}}{\mu_{S}} \nabla p_{S}\right)=0 & \text { in } S, \\ p_{V}=p_{\text {lumen }} & \text { on } \Gamma_{\text {lumen }}, \\ p_{V}=p_{\text {adv }} & \text { on } \Gamma_{\text {adv }}, \\ \frac{k_{V}}{\mu_{V}} \nabla p_{V} \cdot \eta_{V}=0 & \text { on } \Gamma_{\text {wall }}, \\ p_{V}=p_{S} & \text { on } \Gamma_{\text {coat }}, \\ \frac{k_{V}}{\mu_{V}} \nabla p_{V} \cdot \eta_{V}=-\frac{k_{S}}{\mu_{S}} \nabla p_{S} \cdot \eta_{S} & \text { on } \Gamma_{\text {coat }}, \\ \frac{k_{S}}{\mu_{S}} \nabla p_{S} \cdot \eta_{S}=0 & \text { on } \Gamma_{\text {strut }} .\end{cases}
$$

For a sake of simplicity, we assume $p_{\text {adv }}=0$ and a nonzero pressure $p_{\text {lumen }}=p_{0}$.

In what follows we use the notations

$$
\mathcal{A}_{j}\left(p_{j}, q_{j}\right)=\left(\frac{\kappa_{j}}{\mu_{j}} \nabla p_{j}, \nabla q_{j}\right)_{j}, j=S, V,
$$

we use the spaces

$$
H_{\text {lumen }}^{1}(V)=\left\{\vartheta \in H^{1}(V) \text { such that } \vartheta=0 \text { on } \Gamma_{\text {lumen }}\right\}
$$

and

$$
\mathcal{V}=\left\{\left(p_{S}, p_{V}\right) \in H^{1}(S) \times H_{\text {lumen }}^{1}(V) \text { such that } p_{S}=p_{V} \text { on } \Gamma_{\text {coat }}\right\}
$$


Let $w \in H^{1}(V)$ be such that $w=p_{\text {lumen }}$ on $\Gamma_{\text {lumen }}$ and $p_{V}^{*}=p_{V}-w \in H_{\text {lumen }}^{1}(V)$.

With the previous notations, we consider the weak formulation of problem (40):

Find $\left(p_{S}, p_{V}^{*}\right) \in \mathcal{V}$ such that

$$
\mathcal{A}_{S}\left(p_{S}, q_{S}\right)+\mathcal{A}_{V}\left(p_{V}^{*}, q_{V}\right)=-\mathcal{A}_{V}\left(w, q_{V}\right), \quad \forall\left(q_{S}, q_{V}\right) \in \mathcal{V}
$$

It is obvious that $p_{V}$ can be recovered by $p_{V}=p_{V}^{*}+w$.

\section{A.2 Convection-diffusion-reaction problem}

We adopt in what follows the following notations:

$$
\begin{aligned}
& a_{S}\left(v_{S}(t), w_{S}\right)=\sum_{m=1}^{5}\left(D_{m, S} \nabla v_{m, S}(t)-u_{S} v_{m, S}(t), \nabla w_{m, S}\right)_{S}, \\
& a_{V}\left(v_{V}(t), w_{V}\right)=\sum_{\substack{m=1 \\
m \neq 2}}^{5}\left(D_{m, V} \nabla v_{m, V}(t)-u_{V} v_{m, V}(t), \nabla w_{m, V}\right)_{V} \\
&+\sum_{\substack{m=1 \\
m \neq 2}}^{5} \int_{0}^{t} e^{-\frac{t-s}{\tau_{1}}}\left(D_{m, \sigma} \nabla v_{m, V}(s), \nabla w_{m, V}\right)_{V} d s \\
& a_{\text {lumen }}\left(v_{V}(t), w_{V}\right)=\gamma_{1, V}\left(1-v_{1, V}(t), w_{1, V}\right)_{\Gamma_{\text {lumen }}}-\sum_{m=3}^{5} \gamma_{m, V}\left(v_{m, V}(t), w_{m, V}\right)_{\Gamma_{\text {lumen }}} .
\end{aligned}
$$

These bilinear forms are defined in the Sobolev space

$$
\mathcal{W}=\left\{\left(v_{S}, v_{V}\right) \in\left(H^{1}(S)\right)^{5} \times\left(H^{1}(V)\right)^{4} \text { such that } v_{m, S}=v_{m, V} \text { on } \Gamma_{\text {coat }}, m=1,3,4,5\right\}
$$

where $\left(v_{S}, v_{V}\right)=\left(\left(v_{m, S}\right)_{m=1, \ldots, 5},\left(v_{m, V}\right)_{\substack{m=1, \ldots, 5 \\ m \neq 2}}\right)$ and

$$
L^{2}(0, T ; \mathcal{W})=\left\{w:(0, T) \longrightarrow \mathcal{W} \text { such that } \int_{0}^{T}\|w(t)\|_{\mathcal{W}}^{2} d t<\infty\right\}
$$

The weak solution of the problem $(25)-(31)$ is the solution of the following variational problem: Find $\left(\mathcal{C}_{S}, \mathcal{C}_{V}\right) \in L^{2}(0, T, \mathcal{W})$ such that $\left(\frac{\partial \mathcal{C}_{S}}{\partial t}, \frac{\partial \mathcal{C}_{V}}{\partial t}\right) \in\left(L^{2}\left(0, T, L^{2}(S)\right)\right)^{5} \times\left(L^{2}\left(0, T, L^{2}(V)\right)\right)^{4}$ and

$$
\left\{\begin{array}{c}
\sum_{j=S, V}\left(\left(\frac{\partial \mathcal{C}_{j}}{\partial t}(t), v_{j}\right)_{j}+a_{j}\left(\mathcal{C}_{j}(t), v_{j}\right)\right)=\sum_{j=S, V}\left(\mathcal{F}_{j}\left(\mathcal{C}_{j}(t)\right), v_{j}\right)_{j}+a_{\text {lumen }}\left(\mathcal{C}_{V}(t), v_{V}\right) \\
\text { a.e in }(0, T), \text { for all }\left(v_{S}, v_{V}\right) \in \mathcal{W} \\
\mathcal{C}_{S}(0)=(0,1,0,0,1), \mathcal{C}_{V}(0)=(1,0,0,0)
\end{array}\right.
$$

where

$$
\left(\mathcal{F}_{S}\left(\mathcal{C}_{S}\right), \mathcal{F}_{V}\left(\mathcal{C}_{V}\right)\right)=\left(\left(F_{m, S}\left(\mathcal{C}_{S}\right)\right)_{m=1, \ldots, 5},\left(F_{m, V}\left(\mathcal{C}_{V}\right)\right)_{\substack{m=1, \ldots, 5 \\ m \neq 2}}\right)
$$


is defined by (2)-(12).

We define the energy functional

$$
\mathcal{E}(t)=\sum_{j=S, V}\left(\left\|\mathcal{C}_{j}(t)\right\|_{L^{2}(j)}^{2}+\int_{0}^{t}\left\|\nabla \mathcal{C}_{j}(s)\right\|_{L^{2}(j)}^{2} d s\right)+\left\|\int_{0}^{t} e^{-\frac{t-s}{\tau_{1}}} \nabla \mathcal{C}_{V}(s) d s\right\|_{L^{2}(V)}^{2}, \quad t \in[0, T],
$$

where

$$
\left\|\mathcal{C}_{S}(t)\right\|_{L^{2}(S)}=\sum_{m=1}^{5}\left\|C_{m, S}(t)\right\|_{L^{2}(S)} \text { and }\left\|\mathcal{C}_{V}(t)\right\|_{L^{2}(V)}=\sum_{\substack{m=1 \\ m \neq 2}}^{5}\left\|C_{m, V}(t)\right\|_{L^{2}(V)} .
$$

An upper bound for the energy functional (50) is established in the following theorem.

Theorem A.1 If $\left(\mathcal{C}_{S}, \mathcal{C}_{V}\right) \in L^{2}(0, T, \mathcal{W})$, with $\left(\frac{\partial \mathcal{C}_{S}}{\partial t}, \frac{\partial \mathcal{C}_{V}}{\partial t}\right) \in\left(L^{2}\left(0, T ; L^{2}(S)\right)\right)^{5} \times\left(L^{2}\left(0, T ; L^{2}(V)\right)\right)^{4}$, is a solution of the variational problem (48), then assuming $\left(\mathcal{C}_{S}(t), \mathcal{C}_{V}(t)\right) \in\left(H^{2}(S)\right)^{5} \times\left(H^{2}(V)\right)^{4}$ we have

$$
\mathcal{E}(t) \leq \frac{1}{\min \left\{1, \phi, D_{\sigma}\right\}} e^{2(\mathcal{K}+\varphi) t} \mathcal{E}(0)+\frac{\gamma_{1, V}}{2}\left|\Gamma_{\text {lumen }}\right|^{2}\left(e^{2(\mathcal{K}+\varphi) t}-1\right),
$$

where $\mathcal{K}, \phi, \varphi$ and $D_{\sigma}$ are concentration-independent constants while $\left|\Gamma_{\text {lumen }}\right|$ is the length of the transition layer $\Gamma_{\text {lumen }}$.

Estimate (52) proves the stability of the model for finite intervals of time.

\section{B Weak formulation of the discrete model}

We fix $h>0$ and define in $\Omega=S \cup V$ (Figure 2) an admissible triangulation $\mathcal{T}_{h}$, depending on $h>0$, such that the corresponding admissible triangulations in $S$ and $V$, respectively $\mathcal{T}_{h_{S}}$ and $\mathcal{T}_{h_{V}}$, are compatible in $\Gamma_{\text {coat }}$ (see the zoomed part of Figure 4). We represent by $\Delta_{1}$ a typical element of $\mathcal{T}_{h_{S}}$ and by $\Delta_{2}$ a typical element of $\mathcal{T}_{h_{V}}$.

Let $S_{h}=\bigcup_{\Delta_{1} \in \mathcal{T}_{h_{S}}} \Delta_{1}, V_{h}=\bigcup_{\Delta_{2} \in \mathcal{T}_{h_{V}}} \Delta_{2}$ and let $\mathcal{A}_{S, h}(.,$.$) and \mathcal{A}_{V, h}(.,$.$) be defined as \mathcal{A}_{S}(.,$.$) and$ $\mathcal{A}_{V}(.,$.$) but with the L^{2}$ inner product defined on $S_{h}$ and $V_{h}$, respectively. To define the bilinear form corresponding to $a_{\text {lumen }}(.,$.$) , we represent by \Gamma_{\text {lumen }, h}$ the boundary of $V_{h}$ that replaces $\Gamma_{\text {lumen }}$.

We assume that $p_{\text {adv }}=0$ and $p_{\text {lumen }}=p_{0, h}$. We define in what follows the space of globally continuous functions on $S_{h}$ and $V_{h}$ whose restrictions to each element $\Delta_{1}$ and $\Delta_{2}$ respectively, are polynomials of degree at most $n$, i.e.

$$
\begin{aligned}
& \mathcal{V}_{h}=\left\{\left(p_{S, h}, p_{V, h}\right) \in C^{0}\left(\bar{S}_{h}\right) \times C^{0}\left(\bar{V}_{h}\right) \text { such that } p_{S, h}=p_{V, h} \text { on } \Gamma_{\text {coat }} \text { and } p_{V, h}=0 \text { on } \Gamma_{\text {lumen }}\right. \\
& \left.\quad \text { and }\left.\left(p_{S, h}, p_{V, h}\right)\right|_{\Delta_{1} \times \Delta_{2}} \in P_{n} \times P_{n}, \text { for all } \Delta_{1} \in \mathcal{T}_{h_{S}} \text { and } \Delta_{2} \in \mathcal{T}_{h_{V}}\right\} \subset H^{1}\left(S_{h}\right) \times H_{\text {lumen }}^{1}\left(V_{h}\right) .
\end{aligned}
$$

In (53), $P_{n}$ denotes the space of polynomial of degree at most $n$.

The finite dimensional formulation for system (40) reads: 
Find $\left(p_{S, h}, p_{V, h}^{*}\right) \in \mathcal{V}_{h}$ such that

$$
\mathcal{A}_{S, h}\left(p_{S, h}, q_{S, h}\right)+\mathcal{A}_{V, h}\left(p_{V, h}^{*}, q_{V, h}\right)=-\mathcal{A}_{V, h}\left(w_{h}, q_{V, h}\right), \quad \forall\left(q_{S, h}, q_{V, h}\right) \in \mathcal{V}_{h} .
$$

We also use the following notations

$$
\left(v_{S, h}, v_{V, h}\right)=\left(\left(v_{m, S, h}\right)_{m=1, \ldots, 5},\left(v_{m, V, h}\right)_{\substack{m=1, \ldots, 5 \\ m \neq 2}}\right) .
$$

To compute the semi-discrete Ritz-Galerkin approximation $\mathcal{C}_{h}=\left(\mathcal{C}_{S, h}, \mathcal{C}_{V, h}\right)$ for the weak solution of $\mathcal{C}=\left(\mathcal{C}_{S}, \mathcal{C}_{V}\right)$ defined by $(25)-(31)$, we consider the space

$$
\begin{aligned}
\mathcal{W}_{h}= & \left\{\left(v_{S, h}, v_{V, h}\right) \in\left(C^{0}\left(\bar{S}_{h}\right)\right)^{5} \times\left(C^{0}\left(\bar{V}_{h}\right)\right)^{4} \text { such that } v_{m, S, h}=v_{m, V, h} \text { on } \Gamma_{\text {coat }}, m=1,3,4,5\right. \\
& \text { and } \left.\left.\left(v_{S, h}, v_{V, h}\right)\right|_{\Delta_{1} \times \Delta_{2}} \in\left(P_{q}\right)^{5} \times\left(P_{q}\right)^{4} \text { for all } \Delta_{1} \in \mathcal{T}_{h_{S}} \text { and } \Delta_{2} \in \mathcal{T}_{h_{V}}\right\} \subset\left(H^{1}\left(S_{h}\right)\right)^{5} \times\left(H^{1}\left(V_{h}\right)\right)^{4},
\end{aligned}
$$

where $P_{q}$ denotes the space of polynomials of degree at most $q$ (not necessarily equal to $n$ ). By $a_{j, h}(.,$.$) we represent the bilinear form defined on a_{j}(.,$.$) but with the L^{2}$ inner products defined on $S_{h}$ for $j=S$ and $V_{h}$ for $j=V$. By $a_{\text {lumen }, h}(.,$.$) we denote the bilinear form defined as a_{\text {lumen }}(.,$. but considering the boundary integrals on $\Gamma_{\text {lumen }, h}$.

The weak solution of the problem (25) - (31) in the discrete case is the solution of the following finite dimensional variational formulation:

Find $\left(\mathcal{C}_{S, h}, \mathcal{C}_{V, h}\right) \in L^{2}\left(0, T, \mathcal{W}_{h}\right)$, with $\left(\frac{\partial \mathcal{C}_{S, h}}{\partial t}, \frac{\partial \mathcal{C}_{V, h}}{\partial t}\right) \in\left(L^{2}\left(0, T ; L^{2}\left(S_{h}\right)\right)\right)^{5} \times\left(L^{2}\left(0, T ; L^{2}\left(V_{h}\right)\right)\right)^{4}$ and

$$
\left\{\begin{array}{c}
\sum_{j=S, V}\left(\left(\frac{\partial \mathcal{C}_{j, h}}{\partial t}(t), v_{j, h}\right)_{j, h}+a_{j, h}\left(\mathcal{C}_{j, h}(t), v_{j, h}\right)\right)=\sum_{j=S, V}\left(\mathcal{F}_{j}\left(\mathcal{C}_{j, h}(t)\right), v_{j, h}\right)_{j, h}+a_{\text {lumen }, h}\left(\mathcal{C}_{V, h}(t), v_{V, h}\right), \\
\text { a.e in }(0, T), \text { for all }\left(v_{S, h}, v_{V, h}\right) \in \mathcal{W}_{h}, \\
\mathcal{C}_{S, h}(0)=(0,1,0,0,1), \mathcal{C}_{V, h}(0)=(1,0,0,0) .
\end{array}\right.
$$

To conclude this section, we introduce the semi-discrete energy functional

$$
\mathcal{E}_{h}(t)=\sum_{j=S, V}\left(\left\|\mathcal{C}_{j, h}(t)\right\|_{L^{2}(j)}^{2}+\int_{0}^{t}\left\|\nabla \mathcal{C}_{j, h}(s)\right\|_{L^{2}(j)}^{2} d s\right)+\left\|\int_{0}^{t} e^{-\frac{t-s}{\tau_{1}}} \nabla \mathcal{C}_{V, h}(s) d s\right\|_{L^{2}(V)}^{2}, \quad t \in[0, T],(58)
$$

where $\mathcal{C}_{j, h}(t), j=S, V$, is the solution of (57).

This functional is the semi-discrete version of the energy functional (50). Following a procedure analogous to the one in Theorem A.1, a discrete version of inequality (52) can be established.

\section{Validation of the numerical model}

In Table 2 we present the errors

$$
E_{h_{\max }}\left(C_{5, j}\right)=\max _{n=1, \ldots, N}\left\|\bar{C}_{5, j}^{n}-C_{5, j, h}^{n}\right\|_{L^{2}(j)}, j=S, V
$$


where $\bar{C}_{5, j}^{n}$ represents the reference solution computed considering for the stent: $h_{\max }=2.2 \times 10^{-2}$, with 1706 triangles, 1054 vertices; and for the arterial wall: $h_{\max }=4.89 \times 10^{-2}$, with 33033 triangles, 16895 vertices. We also present in this table the convergence rates

$$
R_{h_{1}, h_{2}}\left(C_{5, j}\right)=\frac{\ln \left(\frac{E_{h_{\max }, 1}\left(C_{5, j}\right)}{E_{h_{\max }, 2}\left(C_{5, j}\right)}\right)}{\ln \left(\frac{h_{\max }, 1}{h_{\max }, 2}\right)}, j=S, V,
$$

when $\Delta t$ is fixed, where $h_{\max , 1}$ and $h_{\max , 2}$ are the maximum edge size of triangles of two consecutive generated meshes and $N_{\Delta_{i}}, i=1,2$, are the number of elements used in the triangulation of the stent coating and the arterial wall respectively. We took $\alpha_{m}=1, m=1,3,4,5$.

\begin{tabular}{|c|c|c|c||c|c|c|c|}
\hline$h_{\max }$ & $N_{\Delta_{1}}$ & $E_{h_{\max }}\left(C_{5, S}\right)$ & $R_{h_{1}, h_{2}}\left(C_{5, S}\right)$ & $h_{\max }$ & $N_{\Delta_{2}}$ & $E_{h_{\max }}\left(C_{5, V}\right)$ & $R_{h_{1}, h_{2}}\left(C_{5, V}\right)$ \\
\hline $6.27 \times 10^{-2}$ & 200 & $6.52 \times 10^{-2}$ & - & $1.51 \times 10^{-1}$ & 3688 & $6.56 \times 10^{-2}$ & - \\
$5.10 \times 10^{-2}$ & 298 & $4.72 \times 10^{-2}$ & 1.5728 & $1.27 \times 10^{-1}$ & 5624 & $5.33 \times 10^{-2}$ & 1.1787 \\
$4.39 \times 10^{-2}$ & 454 & $3.59 \times 10^{-2}$ & 1.8435 & $9.87 \times 10^{-2}$ & 8107 & $3.81 \times 10^{-2}$ & 1.3332 \\
$4.09 \times 10^{-2}$ & 522 & $3.11 \times 10^{-2}$ & 1.9925 & $8.49 \times 10^{-2}$ & 10877 & $3.04 \times 10^{-2}$ & 1.4918 \\
$3.27 \times 10^{-2}$ & 786 & $2.00 \times 10^{-2}$ & 1.9823 & $7.92 \times 10^{-2}$ & 14741 & $2.68 \times 10^{-2}$ & 1.8133 \\
$3.00 \times 10^{-2}$ & 1014 & $1.68 \times 10^{-2}$ & 2.0082 & $6.60 \times 10^{-2}$ & 18684 & $1.88 \times 10^{-2}$ & 1.9374 \\
\hline
\end{tabular}

Table 2: Errors and convergence rates for the drug in the stent and arterial wall obtained with $\Delta t=10^{-3}$ and $T=1 h$.

In Table 3 we present the errors $E_{\Delta t k}\left(C_{5, j}\right)$ in $C_{5, j}$ defined by (59) for a fixed triangulation and for different time step sizes and the convergence rates

$$
R_{\Delta t_{1}, \Delta t_{2}}\left(C_{5, j}\right)=\frac{\ln \left(\frac{E_{\Delta t_{1}}\left(C_{5, j}\right)}{E_{\Delta t_{2}}\left(C_{5, j}\right)}\right)}{\ln \left(\frac{\Delta t_{1}}{\Delta t_{2}}\right)}
$$

for $j=S, V$. In (61) $\Delta t_{1}$ and $\Delta t_{2}$ define two consecutive time steps.

\begin{tabular}{|l|c|c||c|c|}
\hline$\Delta t$ & $E_{\Delta t}\left(C_{5, S}\right)$ & $R_{\Delta t_{1}, \Delta t_{2}}\left(C_{5, S}\right)$ & $E_{\Delta t}\left(C_{5, V}\right)$ & $R_{\Delta t_{1}, \Delta t_{2}}\left(C_{5, V}\right)$ \\
\hline 0.01 & $9.91 \times 10^{-2}$ & - & $1.18 \times 10^{-1}$ & - \\
0.075 & $8.73 \times 10^{-2}$ & 0.4365 & $9.86 \times 10^{-2}$ & 0.6321 \\
0.005 & $7.11 \times 10^{-2}$ & 0.5117 & $7.87 \times 10^{-2}$ & 0.5551 \\
0.0025 & $3.98 \times 10^{-2}$ & 0.8351 & $4.55 \times 10^{-2}$ & 0.7921 \\
0.001 & $1.68 \times 10^{-2}$ & 0.9389 & $1.88 \times 10^{-2}$ & 0.9605 \\
\hline
\end{tabular}

Table 3: Errors and convergence rates for the drug obtained with $h_{\max }=3.00 \times 10^{-2}, h_{\max }=$ $6.60 \times 10^{-2}$ for the stent and arterial wall respectively and with $T=1 \mathrm{~h}$.

\section{References}

[1] S.D. Abramowitch, S.D. And S.L. Woo, A new analytical approach to evaluate the viscoelastic properties of the goat medial collateral ligament using the quasilinear viscoelastic theory, Proceedings of the Summer Bioengineering Conference, ASME, Key Biscayne, Florida (2003) 47-92.

[2] G. Acharya And K. Park, Mechanisms of controlled drug release from drug-eluting stents, Adv. Drug Deliver. Rev. 58 (3) (2006) 387-401. 
[3] R. Adams And J. Fournier, Sobolev spaces, Elsevier 2nd edition, 2003.

[4] A. Borghi, E. Foa, R. Balossino, F. Migliavacca and G. Dobini, Modelling drug elution from stents: effect of reversible binding in the vascular wall and degradable polymeric matrix, Comput. Meth. Biomech. Biomed. Eng. 11 (4) (2008) 367-377.

[5] H.F. Brinson And L.C. Brinson, Polymer Engineering Science and Viscoelasticity: An Introduction, Springer, 2010.

[6] L.C. Evans, Partial Differential Equations, Graduate Studies in Mathematics Volume 19, American Mathematical Society, 1998.

[7] J.A. Ferreira, M. Grassi, E. Gudino and P. De Oliveira, A new look to non-Fickian diffusion, Appl. Math. Model. 39 (1) (2015) 194-204.

[8] J.A. Ferreira, M. Grassi, E. Gudino and P. De Oliveira, A 3D model for mechanistic control of drug release, Siam J. Appl. Math. 74 (3) (2014) 620-633.

[9] J.A. Ferreira, J. Naghipoor and P. De Oliveira, Analytical and numerical study of a coupled cardiovascular drug delivery model, J. Comp. Appl. Math. 275 (2015) 433-446.

[10] J.A. Ferreira, J. Naghipoor and P. De Oliveira, The effect of reversible binding sites on the drug release from a drug eluting stent, CMMSE2014(II), I.P. Hamilton and J. VigoAguiar (editors), (2014) 519-530.

[11] Y.C. Fung, Biomechanics: Mechanical Properties of Living Tissues, Springer-Verlag, New York, 1993.

[12] T.C. Gasser, R.W. Ogden And G.A. Holzapfel, Hyperelastic modelling of arterial layers with distributed collagen fibre orientations, J. R. Soc. Interface 3 (2006) 15-35.

[13] B.S. Gow And C.D. Hadfield, The elasticity of canine and human coronary arteries with reference to postmortem changes, Circ. Res. 45 (5) (1979) 588-594.

[14] M. Grassi, G. Pontrelli, L. Teresi, G. Grassi and L.Comel, Novel design of drug delivery in stented arteries: a numerical comparative study, Math. Bios. Eng. 6 (3) (2009) 493-508.

[15] M. Horner, S. Joshi, V. Dhruva, S. Sett and S.F.C. Stewart, A two-species drug delivery model is required to predict deposition from drug-eluting stents, Cardiovasc. Eng. Technol. 1 (3) (2010) 225-234.

[16] T. Khamdaenga, J. Luo, J. Vappou, P. Terdtoon and E.E. Konofagou, Arterial stiffness identification of the human carotid artery using the stress-strain relationship in vivo, Ultrasonic 52 (3) (2012) 402-411.

[17] M. Kohandel, S. Sivaloganathan and G. Tenti, Estimation of the quasi-linear viscoelastic parameters using a genetic algorithm, Math. Comput. Model. 47 (3-4) (2008) 266-270. 
[18] A. Levin, N. Vukmirovic, C.-W Huang and E.R. Edelman, Specific binding to intracellular proteins determines arterial transport properties for rapamycin and paclitaxel, Proc. Natl. Acad. Sci. 101 (2004) 9463-9467.

[19] F. Migliavacca, F. Gervaso, M. Prosi, P. Zunino, S. Minisini, L. Formaggia and G. DuBini, Expansion and drug elution model of a coronary stent, Comp. Meth. Biomech. Biom. Eng. 10 (1) (2007) 63-73.

[20] S. McGinty, A decade of modelling drug release from arterial stents, Math. Biosci. 257 (2014) 80-90.

[21] S. McGinty, S. McKee, R. M. Wadsworth and C. McCormick, Modelling drug-eluting stents, Math. Med. Biol. 28 (2011) 1-29.

[22] A. Muliana and K.R. Rajagopal, Modeling the response of nonlinear viscoelastic biodegradable polymeric stents, Int. J. Solids and Structures 49 (7-8) (2012) 989-1000.

[23] A. Muliana and K.R. Rajagopal, Changes in the response of viscoelastic solids due to changes in their internal structures, Acta Mech. 207 (3-4) (2011) 297-316.

[24] A. Nekouzadeh, K.M. Pryse, E.L. Elson and G.M. Genin, A simplified approach to quasi-linear viscoelastic modeling, J. Biomech. 40 (1) (2007) 3070-3078.

[25] I. Ozolanta, G. Tetere, B. Purinya and V. Kasyanov, Changes in the mechanical properties, biochemical contents and wall structure of the human coronary arteries with age and sex, Med. Eng. Phys. 20 (7) (1998) 523-533.

[26] G. Pontrelli and F. De Monte, Mass diffusion through two-layer porous media: an application to the drug-eluting stent, Int. J. Heat Mass Tran. 50 (17-18) (2007) 3658-3669.

[27] G. Pontrelli and F. De Monte, Modelling of mass dynamics in arterial drug-eluting stents, J. Porous Media 12 (1) (2009) 19-28.

[28] G. Pontrelli And F. De Monte, A multi-layer porous wall model for coronary drug-eluting stents, J. Porous Media 53 (19-20) (2010) 3629-3637.

[29] S. Prabhu And S. Hossainy, Modeling of degradation and drug release from a biodegradable stent coating, J. Biomed. Mater. Res. 50 (3) (2007) 3658-3669.

[30] F. Rossi, T. Casalini, E.R.M. Masi and G. Perale, Bioresorbable polymer coated drug eluting stent: A model study, Mol. Pharm. 9 (7) (2012) 1898-1910.

[31] J. M. Schakenraad, M. J. Hardonk, J. Feijen, I. Molenaar, and P. Nieuwenhuis, Enzymatic activity toward poly(L-lactic acid) implants, J. Biomed. Mater. Res., 24 (5) (1990), $529-545$

[32] P.W. Serruys And A.H. Gershlick, Handbook of Drug eluting stent, CRC Press; 1st edition, 2005.

[33] J. Siepmann and A. Gopferich, Mathematical modeling of bioerodible polymeric drug delivery systems,Adv. Drug Delivery Rev. 48 (2-3) (2001) 229-248. 
[34] J.S. SoARes, Constitutive Modeling for Biodegradable Polymers for Applications in Endovascular Stents, PhD Dissertation, Mechanical Engineering Texas A\&M University, 2008

[35] J.S. Soares, J.E. Moore And K.R. Rajagopal, Modeling of deformation accelerated breakdown of polylactic acid biodegradable stents. ASME J. Med. Sci. 4 (4) (2010) 041007

[36] J.S. Soares And P. Zunino, A mixture model for water uptake, degradation, erosion and drug release from polydisperse polymeric networks. Biomaterials 31 (11) (2010) 3032-3042.

[37] Y. TAO, Nonlinear Viscoelastic Properties and Constitutive Modeling of Blood Vessel, Master Dissertation, Mechanical Engineering National University of Singapore, 2009.

[38] K.L. Troyer, D.J. Estep and C.M. Puttlitz, Viscoelastic effects during loading play an integral role in soft tissue mechanics, Acta Biomat. 8 (1) (2012) 234-243.

[39] A.R. Tzafriri, A. Groothuis, G.S. Price and E.R. Edelman, Stent elution rate determines drug deposition and receptor-mediated effects, J. Controlled Release 161 (2012) 918-926.

[40] C. Vergara and P. Zunino, Multiscale boundary conditions for drug release from cardiovascular stents, Mult. Model Sim. 7 (2) (2008) 565-588.

[41] P. Zunino, Multidimensional pharmacokinetic models applied to the design of drug-eluting stents, Cardiov. Eng. Int. J. 4 (2) (2004) 181-191. 